\title{
Averaging for Diffusions with Fine-Grained Boundaries
}

\author{
James P. Dunyak ${ }^{1,2}$ \\ Department of Mathematics, University of Maryland, College Park, MD 20742, USA
}

Received: 5 August 1993

\begin{abstract}
This article investigates the limiting behavior of a diffusion in a half space with a complicated boundary condition. The boundary condition implements a reflection condition everywhere except a number of small sets or "holes" that meet Dirichlet or mixed boundary conditions. Probabilistic methods associated with the Feynman-Kac formula are used to find the limiting behavior of the diffusion equations as the number of holes gets large and the size of each hole is reduced. With particular scaling homogenization occurs, and we see that the complicated boundary condition is replaced by a simple mixed boundary condition depending on the capacitance and distribution of the holes.
\end{abstract}

\section{Problem Formulation}

Our model problem investigates diffusions in the half space $\mathfrak{R} \times \mathfrak{R} \times \mathfrak{R}^{+}$with a finegrained boundary condition. Our boundary implements Neumann boundary conditions, $\partial u / \partial x_{3}\left(x_{1}, x_{2}, 0\right)=0$, everywhere on $\left\{x: x_{3}=0\right\}$ except at a number of small sets. These exceptional sets are scaled, rotated, and translated copies of a nominal set $H$. On these exceptional sets, Dirichlet conditions, $u\left(x_{1}, x_{2}, 0\right)=0$, or mixed conditions, $\partial u / \partial x_{3}\left(x_{1}, x_{2}, 0\right)-\lambda u\left(x_{1}, x_{2}, 0\right)=0$, occur. We refer to these exceptional sets as "holes," and the number of holes is scaled by $N$ while the size of each hole is scaled by $1 / N$. Figure 1 shows an example of a nominal hole $H$, and Fig. 2 shows a possible boundary configuration with $N=5$. Our goal is to study averaging effects as $N \rightarrow \infty$.

The properties of these collections of small sets are first formally described.

Hole Assumption I. Let $H \subset \mathfrak{R}^{3}$ be a bounded closed set contained in the plane

$$
P \equiv\left\{\left(x_{1}, x_{2}, x_{3}\right): x_{3}=0\right\}
$$

1 This work was partly supported by ARO Grant DAAL03-92-@_0219

2 The author acknowledges the direction and encouragement provided by his advisor, Mark Freidlin 


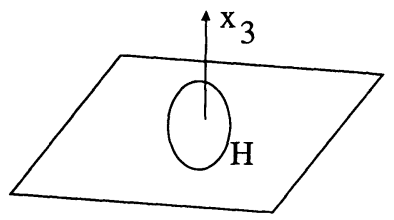

Fig. 1.

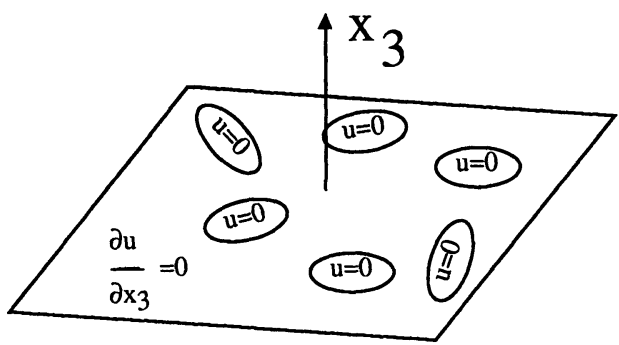

Fig. 2.

with $C^{2}$ boundary in $P$. Assume $(0,0,0) \in H$ and $H \subset\{x:|x| \leq 1\}$. Define, for all $N$ and $i=1,2, \ldots, N$, the collection

$$
H_{i}^{(N)}=\left\{\left(x_{1}, x_{2}, 0\right): N\left(R_{i}^{(N)}\left[\left(x_{1}-y_{1}^{(N)}\right),\left(x_{2}-y_{2}^{(N)}\right)\right],(0)\right) \in H\right\}
$$

for given rotation matrices $R_{i}^{(N)}$ and hole origins $\left(y_{i_{1}}^{(N)}, y_{i_{2}}^{(N)}\right)$.

These collections represent the sets of perturbation to the Neumann boundary condition. Each collection $\left(H_{i}^{(N)}, i=1,2, \ldots, N\right)$ is a set of scaled, rotated, and translated copies of $H$, located on the boundary plane $P$. The holes are scaled in size by $1 / N$ as the number of holes is increased to $N$. Clearly the assumption of the size and location of $H$ are purely for convenience. The proofs below also show that the results are independent of the orientation of the holes. Any set of rotations $R_{i}^{(N)}$ may be used.

We now set up the probability framework. Let $P_{x}$ be the measure on continuous trajectories on $\mathfrak{R} \times \mathfrak{R} \times \mathfrak{R}^{+}$induced by

$$
X_{t}^{x} \equiv\left\{\begin{array}{c}
X_{1_{t}}^{x_{1}} \\
X_{2_{t}}^{x_{2}} \\
X_{3_{t}}^{x_{3}}
\end{array}\right\} \equiv\left\{\begin{array}{c}
W_{1_{t}}^{x_{1}} \\
W_{2_{t}}^{x_{2}} \\
\left|W_{3_{t}}^{x_{3}}\right|
\end{array}\right\}
$$

with $W_{1_{t}}^{x_{1}}, W_{2_{t}}^{x_{2}}, W_{3_{t}}^{x_{3}}$ independent Wiener processes shifted to initial conditions $x$. Note the reflection at the boundary with $x_{3}=0$. The following definitions are also useful:

$$
\begin{aligned}
p_{1}\left(t, x_{1}, y_{1}\right) & =\frac{1}{\sqrt{2 \pi t}} \exp \left\{\frac{-\left(x_{1}-y_{1}\right)^{2}}{2 t}\right\}, \\
p_{2}\left(t, x_{2}, y_{2}\right) & =\frac{1}{\sqrt{2 \pi t}} \exp \left\{\frac{-\left(x_{2}-y_{2}\right)^{2}}{2 t}\right\}, \\
p_{3}\left(t, x_{3}, y_{3}\right) & =\frac{1}{\sqrt{2 \pi t}} \exp \left\{\frac{-\left(x_{3}-y_{3}\right)^{2}}{2 t}\right\}, \\
p(t, x, y) & =p_{1}\left(t, x_{1}, y_{1}\right) p_{2}\left(t, x_{2}, y_{2}\right) p_{3}\left(t, x_{3}, y_{3}\right) .
\end{aligned}
$$

The local time on the boundary is defined as

$$
\zeta_{t}^{x_{3}} \equiv \lim _{\varepsilon \downarrow 0} \frac{1}{2 \varepsilon} \int_{0}^{t} I_{[0, \varepsilon)}\left(X_{3_{t}}^{x_{3}}\right) d s .
$$


The concept of local time on the holes proves useful. For a single hole $H_{i}^{(N)}$, let

$$
{ }^{i} \zeta_{t}^{(N), x_{3}}=\int_{0}^{t} I_{\partial H_{i}^{(N)}}\left(X_{s}^{x}\right) d \zeta_{s}^{x_{3}},
$$

and for the $N^{\text {th }}$ collection,

$$
\zeta_{t}^{(N), x_{3}}=\int_{0}^{t} I_{i=1}^{N} \partial H_{i}^{(N)}\left(X_{s}^{x}\right) d \zeta_{s}^{x_{3}} .
$$
to

Our first goal is to find asymptotic properties of the sequence $u^{(N)}(x, t)$ of solutions

$$
\begin{aligned}
\frac{\partial u^{(N)}}{\partial t} & =\frac{1}{2} \Delta u^{(N)}(x, t) \quad \text { for } t>0, \quad x \in \mathfrak{R} \times \mathfrak{R} \times \mathfrak{R}^{+}, \\
u^{(N)}(x, 0) & =f(x), \\
u^{(N)}(x, t) & \left.=0 \text { for } \quad x \in \bigcup_{i=1}^{N} H_{i}^{(N)} \quad \text { (holes of radius } 1 / N\right), \\
\frac{\partial u^{(N)}}{\partial x_{3}}(x, t) & =0 \text { for } \quad x \in P-\bigcup_{i=1}^{N} H_{i}^{(N)} \quad \text { (insulation), }
\end{aligned}
$$

for $f$ continuously differentiable with compact support. We set by definition the solution to problem (6),

$$
u^{(N)}(x, t)=E_{x}\left\{f\left(X_{t}\right) I_{\sigma^{(N)}>t}\right\}
$$

with

$$
\sigma^{(N)} \equiv\left\{\inf t: X_{t}^{x} \in \bigcup_{i=1}^{N} \partial H_{i}^{(N)}\right\}
$$

We then study the asymptotic limit of

$$
\begin{aligned}
& \frac{\partial u^{(N)}}{\partial t}= \frac{1}{2} \Delta u^{(N)}(x, t) \text { for } t>0, \\
& x \in \mathfrak{R} \times \mathfrak{R} \times \mathfrak{R}^{+} \\
& u^{(N)}(x, 0)=f(x), \\
& \frac{\partial u^{(N)}}{\partial x_{3}}(x, t)-\lambda N^{\gamma} u^{(N)}(x, t)=0 \quad \text { for } \quad x \in \bigcup_{i=1}^{N} H_{i}^{(N)} \quad \text { (holes), } \\
& \frac{\partial u^{(N)}}{\partial x_{3}}(x, t)=0 \quad \text { for } \quad x \in P-\bigcup_{i=1}^{N} H_{\imath}^{(N)} \quad \text { (insulation), }
\end{aligned}
$$

with $\lambda>0, \gamma>0$ and $f$ continuously differentiable with compact support. We set 
by definition the solution to problem (8),

$$
u^{(N)}(x, t)=E_{x}\left\{f\left(X_{t}\right) \exp \left(-\lambda N^{\gamma} \int_{0}^{t} d \zeta_{s}^{(N)}\right)\right\} .
$$

The requirements on initial condition $f$ can be easily relaxed, but are convenient in the proof.

Partial differential equations with boundaries with holes have been widely studied. Most research has been directed towards a Stokes equation called the Neumann Sieve, which describes fluid flow across perforated boundaries with periodic holes. Poisson equations have also been considered. Del Vechio [1], Murat [2] and many others address these problems.

Diffusion equations have also attracted attention. For diffusion equations, most results address holes distributed in three-dimensional regions instead of along boundaries. Kac's paper [3] used the Wiener sausage to establish the limiting effect of spherical, randomly distributed holes with Dirichlet conditions. This was followed by Taylor and Rauch [4] analysis of a similar problem using the same approach. Papanicolaou and Varadhan [5] extended the result to non-spherical holes with both random and nonrandom distributions by use of a semigroup expansion based on the Feynman-Kac formulas. This method is also used in this paper. Figari et al. [6] applied a Green's function approach to achieve similar results. Problems with fine grained boundary conditions are discussed in Khruslov and Marchenko [7], which contains useful discussions on requirements for hole placements.

Our problems are somewhat different. We use probabilistic methods to study how containers "leak," the phenomena by which heat and other processes move from areas of high concentration to areas of zero concentration. We also consider the effects of scaling associated with mixed boundary conditions. Our methods of proof, which provide a strong form of convergence, are motivated by Papanicolaou and Varadhan's paper [5] on diffusions in regions with holes. Although the details here are often quite different, the overall approach and several lemmas from [5] are used. Rauch and Taylor also briefly describe, using analytical methods, diffusions when small spherical holes with Dirichlet conditions are distributed on a smooth surface. Weinryb [8] applied a Wiener sausage approach to circular holes randomly distributed on a boundary.

We will study the behavior of $u^{(N)}(x, t)$, making various assumptions on the distribution of holes. These conditions will be used for both Eqs. (6) and (8).

Hole Assumption II. There exists a bounded, continuous function $V(x) \geq 0, x \in \mathfrak{R}^{2}$, with support contained in a compact set S. For all continuous $\phi$,

$$
\lim _{N \uparrow \infty} \frac{1}{N} \sum_{i=1}^{N} \phi\left(y_{i 1}^{(N)}, y_{i_{2}}^{(N)}\right)=\int_{\mathfrak{R}^{2}} \phi(x) V(x) d x .
$$

Here $\left(y_{i_{1}}^{(N)}, y_{i_{2}}^{(N)}, 0\right)$ is the center of the $i^{\text {th }}$ hole of the $N^{\text {th }}$ collection. For convenience, we further require $H_{i}^{(N)} \subset S$ for all $i$ and $N$, and that $S$ contains the origin.

Hole Assumption III.

$$
\lim _{N \uparrow \infty} \frac{1}{N^{2}} \sum_{\imath, j=1}^{N} \frac{1}{i \neq j}=\iint_{\mathfrak{R}^{2}} \frac{V(x) V(y)}{|x-y|} d x d y .
$$


Hole Assumption III implies a more general relationship. See Lemma 4 of [5]. For any bounded, continuous function $\phi$,

$$
\lim _{N \uparrow \infty} \frac{1}{N^{2}} \sum_{i, j=1}^{N} \frac{\phi\left(y_{i}^{N}, y_{j}^{N}\right)}{\left|y_{\imath}^{N}-y_{j}^{N}\right|}=\iint_{\mathfrak{R}^{2}} \frac{\phi(x, y) V(x) V(y)}{|x-y|} d x d y .
$$

This relationship will be used later.

In [5] we see that both these conditions are met if the holes are located periodically. Theorems I and II below deal with cases in which the hole distributions are deterministic. If the hole centers are independently drawn from a random distribution with density $V$, then Hole Assumption III holds in probability, and Theorems III and IV apply.

The main theorems require several definitions. We first identify the set $D_{\varepsilon}$. Consider a circle $C$ in the plane $P=\left\{x: x_{3}=0\right\}$ with

$$
H_{i}^{(N)} \subset C \text { for all } i \text { and } N \text {, }
$$

and

$$
\operatorname{distance}(P-C, S) \geq 1 \text {. }
$$

Hole Assumption II describes $S$ and implies the existence of such a circle. For fixed $\varepsilon$, choose $\delta$ at least small enough that the set

$$
\begin{aligned}
D_{\varepsilon} \equiv\left\{\left(x_{1}, x_{2}, x_{3}\right): x_{1} \in \mathfrak{R} ; x_{2} \in \mathfrak{R} ;\right. \\
\left.x_{3} \geq \delta \text { if }\left(x_{1}, x_{2}\right) \in C \text { and } x_{3}>0 \text { otherwise }\right\}
\end{aligned}
$$

meets

$$
\operatorname{volume}\left(\mathfrak{R} \times \mathfrak{R} \times \mathfrak{R}^{+}-D_{\varepsilon}\right)<\varepsilon .
$$

Thus $D_{\varepsilon}$ is just $\Re \times \Re \times \Re^{+}$with a thin disk removed. This set $D_{\varepsilon}$ is fixed, after choice of $\varepsilon$, for the remainder of this paper.

We also define two useful parameters. First, associated with the Dirichlet problem (6),

$$
\alpha=\int_{\substack{\text { half sphere } \\ \text { of radius } 1}} w(x) d A
$$

with

$$
\begin{aligned}
& \Delta w=0 \quad x \in \mathfrak{R} \times \mathfrak{R} \times \mathfrak{R}^{+}, \\
& w \rightarrow 0 \text { as }|x| \rightarrow \infty, \\
& \left.w(x)\right|_{x \in H}=1 \text { (hole), } \\
& \left.\frac{\partial w}{\partial x_{3}}(x)\right|_{x \in P-H}=0 \text { (insulation). }
\end{aligned}
$$

This $\alpha$ is just one half the Newtonian capacitance of the hole. See [5] among many other references. For the mixed boundary condition problem, Eq. (8), define

$$
a=\int_{\substack{\text { half sphere } \\ \text { of radius } 1}} v(x) d A
$$


with

$$
\begin{aligned}
& \Delta v=0 \quad x \in \mathfrak{R} \times \mathfrak{R} \times \mathfrak{R}^{+}, \\
& v \rightarrow 0 \text { as }|x| \rightarrow \infty \\
& \frac{\partial v}{\partial x_{3}}(x)-\left.\lambda v(x)\right|_{x \in H}=-\lambda \text { (hole), } \\
& \left.\frac{\partial v}{\partial x_{3}}(x)\right|_{x \in P-H}=0 \text { (insulation). }
\end{aligned}
$$

The constant $a$, determined by both hole shape and $\lambda$, plays a role similar to capacitance.

We can now turn to the main theorems.

Theorem I. Assume Hole Assumptions I, II, and III are met. Let $\varepsilon>0$ and $T<\infty$. Define

$$
u^{(N)}(x, t)=E_{x}\left\{f\left(X_{t}\right) I_{\sigma^{(N)}>t}\right\}
$$

with

$$
\sigma^{(N)} \equiv\left\{\inf t: X_{t}^{x} \in \bigcup_{i=1}^{N} \partial H_{i}^{(N)}\right\}
$$

and assume $f$ is continuously differentiable with compact support. Then there exists an integer $N_{0}(\varepsilon, T)$ and $D_{\varepsilon}$ (defined above) such that, for all $N \geq N_{0}(\varepsilon, T)$,

$$
\sup _{0 \leq t \leq T} \sup _{x \in D_{\varepsilon}}\left|u^{(N)}(x, t)-u(x, t)\right|<\varepsilon
$$

with

$$
\operatorname{volume}\left(\Re \times \Re \times \Re^{+}-D_{\varepsilon}\right)<\varepsilon
$$

and

$$
u(x, t)=E_{x}\left\{f\left(X_{t}\right) \exp \left(-2 \alpha \int_{0}^{t} V\left(X_{1_{s}}, X_{2_{s}}\right) d \zeta_{s}\right)\right\}
$$

$u$ is the solution of

$$
\begin{gathered}
\frac{\partial u}{\partial t}=\frac{1}{2} \Delta u(x, t) \quad \text { for } t \geq 0, x \in \mathfrak{R} \times \mathfrak{R} \times \mathfrak{R}^{+}, \\
u(x, 0)=f(x), \\
{\left[\frac{\partial u}{\partial x_{3}}(x, t)-2 \alpha V\left(x_{1}, x_{2}\right) u(x, t)\right]_{x \in\left\{x_{3}=0\right\}}=0 .}
\end{gathered}
$$

This theorem has a transparent interpretation in the probabilistic setting. The contribution of each trajectory to the expectation is reduced by the local time on the boundary.

Theorem II illustrates the effect of the constant $\gamma$ on the mixed boundary condition. 
Theorem II. Assume Hole Assumptions I, II, and III are met. Let $\varepsilon>0, \gamma>0$, $\lambda>0$, and $T<\infty$. Define

with

$$
u^{(N)}(x, t)=E_{x}\left\{f\left(X_{t}\right) \exp \left(-\lambda N^{\gamma} \int_{0}^{t} d \zeta_{s}^{(N)}\right)\right\}
$$

$$
\zeta_{t}^{(N), x_{3}}=\int_{0}^{t} I_{\bigcup_{i=1}^{N} \partial H_{i}^{(N)}}\left(X_{s}^{x}\right) d \zeta_{3}^{x_{3}}
$$

and assume $f$ is continuously differentiable with compact support. Then there exists an integer $N_{0}(\varepsilon, T)$ and $D_{\varepsilon}$ (defined above) such that, for all $N \geq N_{0}(\varepsilon, T)$,

$$
\sup _{0 \leq t \leq T} \sup _{x \in D_{\varepsilon}}\left|u^{(N)}(x, t)-u(x, t)\right|<\varepsilon
$$

with

$$
\operatorname{volume}\left(\mathfrak{R} \times \mathfrak{R} \times \mathfrak{R}^{+}-D_{\varepsilon}\right)<\varepsilon
$$

and

$$
u(x, t)=E_{x}\left\{f\left(X_{t}\right) \exp \left(-2 a(\gamma) \int_{0}^{t} V\left(X_{1_{s}}, X_{2_{s}}\right) d \zeta_{s}\right)\right\}
$$

$u$ is the solution of

$$
\begin{gathered}
\frac{\partial u}{\partial t}=\frac{1}{2} \Delta u(x, t) \text { for } t \geq 0, x \in \mathfrak{R} \times \mathfrak{R} \times \mathfrak{R}^{+} \\
u(x, t)=f(x) \\
{\left[\frac{\partial u}{\partial x_{3}}(x, t)-2 a(\gamma) V\left(x_{1}, x_{2}\right) u(x, t)\right]_{x \in\left\{x_{3}=0\right\}}=0 .}
\end{gathered}
$$

Here

$$
a(\gamma)=\left\{\begin{array}{lll}
0 & \text { for } & \gamma<1 \\
a & \text { for } & \gamma=1 \\
\alpha & \text { for } & \gamma>1
\end{array}\right.
$$

Again the theorem has a clear interpretation in Eq. (16). We see the critical value is $\gamma=1$. For $\gamma<1$, the effect of the holes disappears. For $\gamma=1$, the scaling is appropriate for boundary conditions resulting from a thin layer, in which the layer thickness scales linearly with the diameter of the hole. For $\gamma>1$, the mixed boundary condition is dominated by the non-derivative term, and the same limiting behavior as problem (6) occurs.

Theorems I and II apply to deterministic hole distributions meeting Eqs. (9) and (10). Random hole distributions may also be considered using the viewpoint in [5]. If each $y_{l}^{N}$ is an independent draw from a random distribution with density $V$, then Eq. (10) holds in probability. Consider the probability space $\left(\Omega^{N}, F^{N}, P^{N}\right)$ associated with the $N^{\text {th }}$ hole distribution. In this case, $u^{(N)}(x, t)=u^{(N)}(x, t, \omega)$ for $\omega \in \Omega^{N}$. Theorems I and II then become: 
Theorem III. Let the hole centers $y_{2}^{(N)}$ be independent and identically distributed with density $V(x)$. Assume Hole Assumptions I, II, and III are met, with Hole Assumption III holding in probability. Let $\varepsilon>0, \delta>0$ and $T<\infty$. Define

$$
u^{(N)}(x, t, \omega)=E_{x, \omega}\left\{f\left(X_{t}\right) I_{\sigma^{(N)}>t}\right\}
$$

with

$$
\sigma^{(N)} \equiv\left\{\inf t: X_{t}^{x} \in \bigcup_{\imath=1}^{N} \partial H_{\imath}^{(N)}\right\}
$$

and assume $f$ is continuously differentiable with compact support. Then there exists an integer $N_{0}(\varepsilon, T)$, sets $\Omega_{\varepsilon, \delta}^{N} \subset \Omega^{N}$, and $D_{\varepsilon}$ (defined above) such that, for all $N \geq N_{0}(\varepsilon, T)$,

$$
\sup _{0 \leq t \leq T} \sup _{x \in D_{\varepsilon}}\left|u^{(N)}(x, t, \omega)-u(x, t)\right|<\varepsilon, \quad \text { when } \omega \in \Omega_{\varepsilon, \delta}^{N}
$$

with

$$
\begin{array}{r}
\text { volume }\left(\Re \times \Re \times \Re^{+}-D_{\varepsilon}\right)<\varepsilon \\
\text { Probability }\left(\Omega^{N}-\Omega_{\varepsilon, \delta}^{N}\right)<\delta
\end{array}
$$

and

$$
u(x, t)=E_{x}\left\{f\left(X_{t}\right) \exp \left(-2 \alpha \int_{0}^{t} V\left(X_{1_{s}}, X_{2_{s}}\right) d \zeta_{s}\right)\right\}
$$

$u$ is the solution of

$$
\begin{gathered}
\frac{\partial u}{\partial t}=\frac{1}{2} \Delta u(x, t) \quad \text { for } \quad t \geq 0, x \in \Re \times \Re \times \mathfrak{R}^{+}, \\
u(x, 0)=f(x), \\
{\left[\frac{\partial u}{\partial x_{3}}(x, t)-2 \alpha V\left(x_{1}, x_{2}\right) u(x, t)\right]_{x \in\left\{x_{3}=0\right\}}=0 .}
\end{gathered}
$$

Theorem IV. Let the hole centers $y_{2}^{(N)}$ be independent and identically distributed with density $V(x)$. Assume Hole Assumptions I, II, and III are met, with Hole Assumption III holding in probability. Let $\varepsilon>0, \delta>0, \gamma>0, \lambda>0$ and $T<\infty$. Define

$$
u^{(N)}(x, t, \omega)=E_{x, \omega}\left\{f\left(X_{t}\right) \exp \left(-\lambda N^{\gamma} \int_{0}^{t} d \zeta_{s}^{(N)}\right)\right\} .
$$

with

$$
\zeta_{t}^{(N), x_{3}}=\int_{0}^{t} I_{\bigcup_{i=1}^{N} \partial \dot{H}_{i}^{(N)}}\left(X_{s}^{x}\right) d \zeta_{s}^{x_{3}}
$$

and assume $f$ is continuously differentiable with compact support. Then there exists an integer $N_{0}(\varepsilon, T)$, sets $\Omega_{\varepsilon, \delta}^{N} \subset \Omega^{N}$, and $D_{\varepsilon}$ (defined above) such that, for all 
$N \geq N_{0}(\varepsilon, T)$

$$
\sup _{0 \leq t \leq T} \sup _{x \in D_{\varepsilon}}\left|u^{(N)}(x, t, \omega)-u(x, t)\right|<\varepsilon, \quad \text { when } \omega \in \Omega_{\varepsilon, \delta}^{N}
$$

with

$$
\begin{array}{r}
\operatorname{volume}\left(\Re \times \Re \times \Re^{+}-D_{\varepsilon}\right)<\varepsilon \\
\text { Probability }\left(\Omega^{N}-\Omega_{\varepsilon, \delta}^{N}\right)<\delta
\end{array}
$$

and

$$
u(x, t)=E_{x}\left\{f\left(X_{t}\right) \exp \left(-2 a(\gamma) \int_{0}^{t} V\left(X_{1_{s}}, X_{2_{s}}\right) d \zeta_{s}\right)\right\} .
$$

$u$ is the solution of

$$
\begin{gathered}
\frac{\partial u}{\partial t}=\frac{1}{2} \Delta u(x, t) \quad \text { for } \quad t \geq 0, x \in \mathfrak{R} \times \mathfrak{R} \times \mathfrak{R}^{+} \\
u(x, 0)=f(x), \\
{\left[\frac{\partial u}{\partial x_{3}}(x, t)-2 a(\gamma) V\left(x_{1}, x_{2}\right) u(x, t)\right]_{x \in\left\{x_{3}=0\right\}}=0 .}
\end{gathered}
$$

Here,

$$
a(\gamma)=\left\{\begin{array}{lll}
0 & \text { for } & \gamma<1 \\
a & \text { for } & \gamma=1 \\
\alpha & \text { for } & \gamma>1
\end{array}\right.
$$

The proofs of Theorems III and IV essentially follow from Theorems I and II, with set $\Omega_{\varepsilon, \delta}^{N}$ chosen so that the difference in (10) is sufficiently small. We provide a detailed proof of Theorem I, starting with a series of lemmas in the next section and the proof in the section after that. The proof of Theorem II is then summarized by sketching the differences with the proof of Theorem I.

\section{Supporting Lemmas for Theorem I}

In this section, only the Dirichlet problem in Eq. (6) is considered.

We first make estimates for the case of a single hole scaled in size by $N$. The stopping time for hitting the hole centered at $y$ of size $1 / N$ is

$$
\sigma_{y}^{(N)} \equiv \inf \left[t: X_{t}^{x} \in\left\{\left(x_{1}, x_{2}, x_{3}\right):\left(N\left(x_{1}-y_{1}\right), N\left(x_{2}-y_{2}\right), N x_{3}\right) \in H\right\}\right] .
$$

Lemma 1. For $T>0, \varepsilon>0, D$ a compact set not intersecting $S$,

$$
N P_{x}\left[\sigma_{y}^{(N)} \leq t\right] \rightarrow 2 \alpha \int_{o}^{t} p(s, x, y) d s \quad \text { as } \quad N \rightarrow \infty
$$

uniformly for $x \in D, y \in S$, and $0 \leq t \leq T$. This result is independent of the rotational orientation of the hole. 
Fig. 3.

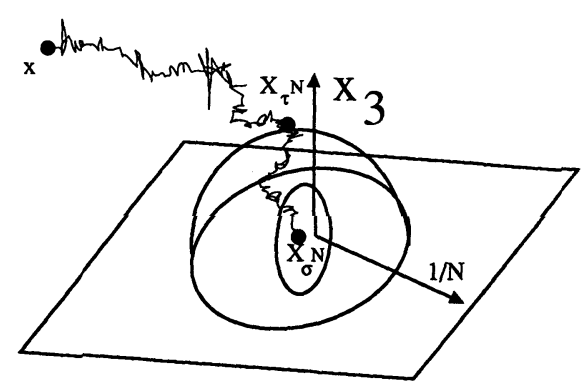

Proof. Without loss of generality, assume $y=0$. Define

$$
\begin{aligned}
\tau^{(N)} & \equiv \inf \left[t:\left|X_{t}^{x}\right|=\frac{1}{N}\right] \\
X_{\tau^{(N)}} & \equiv\left\{\begin{array}{ccc}
X_{\tau^{(N)}} & \text { if } & \tau^{(N)}<\infty \\
0 & \text { if } & \tau_{(N)}=\infty
\end{array}\right\} .
\end{aligned}
$$

Note $\tau^{N} \leq \sigma^{N}$ because we have assumed the hole $H$ fits into a ball of radius one. Then

$$
\begin{aligned}
\lim _{N \rightarrow \infty} E_{x}\left[N I_{\sigma_{0}^{(N)} \leq t}\right]= & \lim _{N \rightarrow \infty} E_{x}\left[N I_{\sigma_{0}^{(N)} \leq t} I_{\tau^{(N)} \leq t}\right] \\
= & \lim _{N \rightarrow \infty} E_{x}\left[N I_{\sigma_{0}^{(N)}<\infty} I_{\tau^{(N)} \leq t}\right] \\
& -\lim _{N \rightarrow \infty} E_{x}\left[N I_{t<\sigma_{0}^{(N)}<\infty} I_{\tau^{(N)} \leq t}\right] .
\end{aligned}
$$

The two terms of Eq. (21) are dealt with separately, with the first term providing the needed bound, and the second going to zero.

We first describe the proof method heuristically. The approach used here is illustrated in Fig. 3. A random path is followed until it intersects a sphere of radius $1 / N$ at time $\tau^{(N)}$. We then expect, for large $N$, that a path hitting the hole will hit the hole quickly. Thus term one of (21) should provide the needed result, and the second term in (21) should be zero. For large $N$, we also expect $X_{\tau^{(N)}}$ to almost uniformly distributed over the sphere of radius $1 / N$. This convergence to uniform distribution implies hole rotations $R_{i}^{N}$ make no contribution. We now present the details of the argument.

Note that for large $N$, the distribution of $X_{\tau^{(N)}}$, conditioned on $\tau^{(N)}<\infty$, converges to uniform on the half sphere of radius $1 / N$. Thus we use for term one of (21)

$$
\begin{aligned}
& \lim _{N \rightarrow \infty} E_{x}\left[N I_{\sigma_{0}^{(N)}<\infty} I_{\tau^{(N)} \leq t}\right] \\
& \quad=\lim _{N \rightarrow \infty} E_{x}\left[N I_{\tau^{(N)} \leq t} E\left(I_{\sigma_{0}^{(N)}<\infty} \mid X_{\tau^{(N)}}, \tau^{(N)}, I_{\tau^{(N)}<\infty}\right)\right]
\end{aligned}
$$


The conditional expectation is calculated in the limit by rescaling $\hat{x}=N x$. Use the definition of $\alpha$ and, from Lemma 1 of [5],

$$
N E_{x}\left[I_{\tau^{(N)} \leq t}\right] \rightarrow 4 \pi \int_{0}^{t} p(s, x, 0) d s \quad \text { as } \quad N \rightarrow \infty
$$

with

$$
p(t, x, y)=\frac{1}{(2 \pi t)^{3 / 2}} \exp \left\{\frac{-|x-y|^{2}}{2 t}\right\} .
$$

Straightforward calculation then yields

$$
\lim _{N \rightarrow \infty} E_{x}\left[N I_{\sigma_{0}^{(N)}<\infty} I_{\tau^{(N)} \leq t}\right]=2 \alpha \int_{0}^{t} p(s, x, 0) d s .
$$

To finish the proof of Lemma 1, we need only show that the second term of (21) goes to zero. Consider a $\Delta$, to be chosen later, with $0 \leq \Delta \leq t$. Then, conditioning on the time and location of the first hitting of the sphere of radius $1 / N$,

$$
\begin{aligned}
& \lim _{N \rightarrow \infty} E_{x}\left[N I_{t<\sigma_{0}^{(N)}<\infty} I_{\tau^{(N)} \leq t}\right] \\
& \quad \leq \lim _{N \rightarrow \infty} E_{x}\left[N I_{i-\Delta<\tau^{(N)} \leq t}\right] \\
& \quad+\lim _{N \rightarrow \infty} E_{x}\left[N I_{\tau^{(N)} \leq t-\Delta} E\left(I_{t<\sigma_{0}^{(N)}<\infty} \mid X_{\tau^{(N)}}, \tau^{(N)}, I_{\tau^{(N)} \leq t-\Delta}\right)\right] .
\end{aligned}
$$

Recalling that $\tau^{(N)}$ is the stopping time for hitting the sphere of radius $1 / N$, the first term may be made as small as desired via choice of $\Delta$ and $N$. This is clear from Eq. (22). Limiting the second term is accomplished by examining the conditional expectation. By rescaling $\hat{t}=N^{2} t$ and $\hat{x}=N x$, as we did above, we immediately see that the calculation of the limit may be reduced to

$$
\lim _{N \rightarrow \infty} \sup _{|x|=1} P_{x}\left\{\Delta N^{2}<\inf \left\{t: X_{t} \in H\right\}<\infty\right\}=0 .
$$

Uniformity in $t \in[0, T], y \in S$, and $x \in D$ results form compactness and continuity. QED.

The second lemma is a simple result to bound solution at large $|x|$.

Lemma 2. For $T>0$ and $\varepsilon>0$, there exists an $r(\varepsilon, T)$ and $N_{0}(\varepsilon, T)$ with the following property. For all

$$
\begin{gathered}
x \in \mathfrak{R} \times \mathfrak{R} \times \mathfrak{R}^{+} \quad \text { with }|x| \geq r, N \geq N_{0}, \quad \text { and } 0 \leq t \leq T, \\
\quad N P_{x}\left\{\sigma_{y_{i}^{(N)}}^{(N)} \leq t\right\}<\varepsilon .
\end{gathered}
$$

Proof. Recall the holes are located in a bounded set. Without loss of generality, presume $y_{i}^{(N)}=0$. Define again

$$
\tau^{(N)} \equiv\left[\inf t:\left|X_{t}^{x}\right|=\frac{1}{N}\right]
$$


We can, via a partial differential equation, solve explicitly for

$$
N P_{x}\left\{\tau_{0}^{(N)}<\infty\right\}=\frac{1}{|x|} \rightarrow 0 .
$$

Note $\tau_{0}^{(N)} \leq \sigma_{0}^{(N)}$, so the result follows when $|x| \rightarrow \infty$. QED.

The third lemma is one side of the convergence for collections of holes.

Lemma 3. Given $\varepsilon>0, T<\infty$, and closed $D \subset \mathfrak{R} \times \mathfrak{R} \times \mathfrak{R}^{+}$not intersecting $S$, there exists an $N_{0}(\varepsilon, T)$ with the following. For all

$$
\begin{gathered}
N \geq N_{0}, \quad x \in D, \quad \text { and } \quad 0 \leq t \leq T \\
P_{x}\left\{\sigma^{(N)} \leq t\right\}<2 E_{x}\left[\alpha \int_{0}^{t} V\left(X_{s}^{1}, X_{s}^{2}\right) d \zeta_{s}\right]+\varepsilon
\end{gathered}
$$

with

$$
\sigma^{(N)} \equiv\left\{\inf t: X_{t}^{x} \in \bigcup_{i=1}^{N} \partial H_{i}^{(N)}\right\}
$$

Proof. Note that

$$
P_{x}\left\{\sigma^{(N)} \leq t\right\} \leq \sum_{i=1}^{N} P_{x}\left\{\sigma_{y_{i}^{(N)}}^{(N)} \leq t\right\} .
$$

We split $D$ into two portions: a far from the origin subset handled by Lemma 2 , and a compact, close to the origin subset handled by Lemma 1.

First the far from the origin subset is handled. Note from Lemma 2 that there exists an $r(\varepsilon, T)$ such that, if $|x|>r$ and $t \leq T$, then

$$
\sum_{i=1}^{N} P_{x}\left\{\sigma_{y_{i}^{(N)}}^{(N)} \leq t\right\}<\varepsilon
$$

We use this $r$ to define compact $\hat{D} \equiv D \cap\{|x| \leq r\}$.

From Lemma 1,

$$
N P_{x}\left[\sigma_{y}^{(N)} \leq t\right] \rightarrow 2 \alpha \int_{0}^{t} p(s, x, y) d s
$$

uniformly for $x \in \hat{D}, y \in S$, and $0 \leq t \leq T$. We conclude there exists an $N_{1}(\varepsilon, T)$ such that

$$
\left|\frac{1}{N} \sum_{i=1}^{N} N P_{x}\left\{\sigma_{y_{\imath}^{(N)}}^{(N)} \leq t\right\}-\frac{1}{N} \sum_{\imath=1}^{N} 2 \alpha \int_{0}^{t} p\left(s, x, y_{\imath}^{(N)}\right) d s\right|<\frac{\varepsilon}{2}
$$

for all $N \geq N_{1}, x \in \hat{D}$ and $0 \leq t \leq T$. Using Assumption II on hole spacing, we see there exists an $N_{2}(\varepsilon, T)$ such

$$
\left|\sum_{i=1}^{N} P_{x}\left\{\sigma_{y_{\imath}^{(N)}}^{(N)} \leq t\right\}-\int_{\mathfrak{R}^{2}} 2 \alpha\left(\int_{0}^{t} p(s, x, y) d s\right) V\left(y_{1}, y_{2}\right) d y_{1} d y_{2}\right|<\frac{\varepsilon}{2}
$$


for all $N \geq N_{2}, x \in \widehat{D}$ and $0 \leq t \leq T$. We now examine the integral by using expression (23) for $p$ and Fubini's theorem. Identify

$$
\begin{gathered}
E_{x_{1} x_{2}}\left[V\left(X_{1_{s}}, X_{2_{s}}\right)\right]=\int_{S} p_{1}\left(s, x_{1}, y_{1}\right) p_{2}\left(s, x_{2}, y_{2}\right) V\left(y_{1}, y_{2}\right) d y_{1} d y_{2} \\
\left|\sum_{\imath=1}^{N} P_{x}\left\{\sigma_{y_{\imath}^{(N)}}^{(N)} \leq t\right\}-2 \alpha \int_{0}^{t} E_{x_{1} x_{2}}\left[V\left(X_{1_{s}}, X_{2_{s}}\right)\right] p_{3}\left(s, x_{3}, 0\right) d s\right|<\frac{\varepsilon}{2} .
\end{gathered}
$$

The integral now may be evaluated using a simple result on local time on the boundary, whose proof is deferred to Lemma 4 . We have

$$
\left|\sum_{i=1}^{N} P_{x}\left\{\sigma_{y_{i}^{(N)}}^{(N)} \leq t\right\}-2 E_{x}\left(\alpha \int_{0}^{t} E_{x_{1} x_{2}}\left[V\left(X_{1_{s}}, X_{2_{s}}\right)\right] d \zeta_{s}\right)\right|<\frac{\varepsilon}{2}
$$

and use independence of $X_{1_{s}}, X_{2_{s}}$, and $X_{3_{s}}$ to see

$$
\left|\sum_{i=1}^{N} P_{x}\left\{\sigma_{y_{i}^{(N)}}^{(N)} \leq t\right\}-2 E_{x}\left(\alpha \int_{0}^{t} V\left(X_{1_{s}}, X_{2_{s}}\right) d \zeta_{s}\right)\right|<\varepsilon
$$

Only Lemma 4 remains to be shown. QED.

Lemma 4 is a simple result on integrals with respect to local time.

Lemma 4. For one-dimensional Brownian motion with reflection at 0 and continuous function $F$,

$$
E_{x}\left[\int_{0}^{t} F(s) d \zeta_{s}\right]=\int_{0}^{t} F(s) p_{3}(s, x, 0) d s
$$

Proof. Note that $E_{x} \zeta_{t}$ is the solution to

$$
\begin{gathered}
\frac{\partial u}{\partial t}=\frac{1}{2} \Delta u(x, t) \text { for } t \geq 0, x \in \mathfrak{R}^{+}, \\
u(x, 0)=0, \\
\left.\frac{\partial u}{\partial x}(x, t)\right|_{x=0}=-1 .
\end{gathered}
$$

Direct substitution establishes

$$
\int_{0}^{t} p_{3}(s, x, 0) d s
$$

is a solution. The lemma follows by considering simple functions $F i(s) \uparrow F(s)$. QED. 
Lemma 5. Given $\varepsilon>0, T<\infty$, and closed $D \subset \mathfrak{R} \times \mathfrak{R} \times \mathfrak{R}^{+}$not intersecting $S$, there exists an $N_{0}(\varepsilon, T)$ with the following. Let

$$
d=\frac{1}{2} \operatorname{distance}(D, S) \text {. }
$$

For all $N \geq N_{0}, x \in D$, and $0 \leq t \leq T$,

$$
P_{x}\left\{\sigma^{(N)} \leq t\right\}>2 E_{x}\left[\alpha \int_{0}^{t} V\left(X_{s}^{1}, X_{s}^{2}\right) d \zeta_{s}\right]-\varepsilon-g(t)
$$

with

$$
\begin{aligned}
g(t) & =t C \exp \left(\frac{-d}{2 t}\right) \text { for a constant } C, \\
\sigma^{(N)} & \equiv \inf \left\{t: X_{t}^{x} \in \bigcup_{i=1}^{N} \partial H_{i}^{(N)}\right\} .
\end{aligned}
$$

The constant $C$ is independent of $N, \varepsilon, D$, and $T$.

Proof. We need only consider compact $D$ since, because of the finite support of $V$,

$$
\sup _{|x|>R} \sup _{0 \leq t \leq T} P_{x}\left\{\sigma^{(N)} \leq t\right\}
$$

and

$$
\sup _{|x|>R} \sup _{0 \leq t \leq T} 2 E_{x}\left[\alpha \int_{0}^{t} V\left(X_{s}^{1}, X_{s}^{2}\right) d \zeta_{s}\right]
$$

are small for large enough $R$.

Let $\varepsilon>0$. We first consider the contributions from holes that are adequately far apart. We sum over all holes whose centers are at least $3 / N$ from their nearest neighbor. Let $\hat{\sigma}^{(N)}$ be the minimum of $\sigma_{y_{i}^{(N)}}^{(N)}$ for $\left\{i:\left|y_{i}^{(N)}-y_{j}^{(N)}\right| \geq 3 / N \forall j \neq i\right\}$. Using inclusion/exclusion, and noting the first summation is over $i$ and the second over $i$ and $j$,

$$
\begin{aligned}
P_{x}\left\{\sigma^{(N)} \leq t\right\} \geq & P_{x}\left\{\hat{\sigma}^{(N)} \leq t\right\} \\
\geq & \sum_{i:\left[\left|y_{\imath}^{(N)}-y_{j}^{(N)}\right| \geq 3 / N \quad \forall j \neq \imath\right]} P_{x}\left\{\sigma_{y_{i}^{(N)}}^{(N)} \leq t\right\} \\
& -\sum_{i, j:\left[\left|y_{i}^{(N)}-y_{j}^{(N)}\right| \geq 3 / N \quad \forall i \neq j\right]} P_{x}\left\{\sigma_{y_{i}^{(N)}}^{(N)} \leq t, \sigma_{y_{j}^{(N)}}^{(N)} \leq t\right\} .
\end{aligned}
$$

The first term of (27) is handled first. Note

$$
\begin{aligned}
& \left.\sum_{i:\left[\left|y_{i}^{(N)}-y_{j}^{(N)}\right| \geq 3 / N\right.} \forall j \neq i\right] \\
& =\sum_{i=1}^{N} P_{x}\left\{\sigma_{y_{i}^{(N)}}^{(N)} \leq t\right\} \\
& \left.\sigma_{i}^{(N)} \leq t\right\}-\sum_{i:\left[\left|y_{i}^{(N)}-y_{j}^{(N)}\right|<3 / N \text { for some } \jmath \neq i\right]} P_{x}\left\{\sigma_{y_{i}^{(N)}}^{(N)} \leq t\right\} .
\end{aligned}
$$




\section{Fig. 4.}



The first summation is handled as in the proof of Lemma 3. The second summation takes a moment of consideration. Since the choice of regions specifically separates the starting point $x$ from the holes, we can call the minimum separation $d$. Now we consider $\gamma>3 / N$, to be chosen later, and two smooth functions $\phi_{d}(s) \geq 0$ and $\psi_{\gamma}(s) \geq 0$ with $\psi_{\gamma}(s)=1$ for $s<\gamma$, and $\psi_{\gamma}(s)=0$ for $s>2 \gamma$. We also require $\phi_{d}(s) \geq I_{s>d}$ with $\phi_{d}(s)=1$ for $s>d$, and $\phi_{d}(s)=0$ for $s<d / 2$. Figure 4 shows two candidate functions. Consider some $\hat{i}$ and $\hat{j}$ such that $\left|y_{\hat{i}}^{(N)}-y_{\hat{j}}^{(N)}\right|<3 / N$. Then obviously

$$
1 \leq \frac{3}{N\left|y_{\hat{i}}^{(N)}-y_{\hat{j}}^{(N)}\right|} \leq \frac{3 \psi_{\gamma}\left(\left|y_{\hat{j}}^{(N)}-y_{\hat{\imath}}^{(N)}\right|\right)}{N\left|y_{\hat{j}}^{(N)}-y_{\hat{i}}^{(N)}\right|}
$$

Again define

$$
\tau_{y_{i}^{(N)}}^{(N)}=\inf \left\{t:\left|X_{t}^{x}-y_{i}^{(N)}\right|=\frac{1}{N}\right\}
$$

and note $\sigma_{y_{\imath}^{(N)}}^{(N)} \geq \tau_{y_{\imath}^{(N)}}^{(N)}$. The relation in Eq. (24) leads immediately to

$$
P_{x}\left\{\sigma_{y_{\imath}^{(N)}}^{(N)} \leq t\right\} \leq \frac{\phi_{d}\left(\left|x-y_{i}^{(N)}\right|\right)}{N\left|x-y_{i}^{(N)}\right|}
$$

We then know from Eqs. (28) and (29), going to a double summation by adding many extra non-negative terms to a summation, that

$$
\begin{gathered}
\sum_{i:\left[\left|y_{i}^{(N)}-y_{\jmath}^{(N)}\right|<3 / N \text { for some } \jmath \neq \imath\right]} P_{x}\left\{\sigma_{y_{i}^{(N)}}^{(N)} \leq t\right\} . \\
\leq \frac{3}{N^{2}} \sum_{\imath \neq \jmath} \frac{\phi_{d}\left(\left|x-y_{\imath}^{(N)}\right|\right)}{\left|x-y_{\imath}^{(N)}\right|} \frac{\psi_{\gamma}\left(\left|y_{\jmath}^{(N)}-y_{\imath}^{(N)}\right|\right)}{\left|y_{\jmath}^{(N)}-y_{i}^{(N)}\right|}
\end{gathered}
$$

for large enough $N$. Using Hole Assumption III and Eq. (11), we see the term converges uniformly in $x$ to

$$
3 \iint \frac{\phi_{d}(|x-z|)}{|x-z|} \frac{\psi_{\gamma}(|z-\hat{z}|)}{|z-\hat{z}|} V(z) V(\hat{z}) d z d \hat{z} \quad \text { as } \quad N \rightarrow \infty \text {. }
$$


The double integral is then analyzed using polar coordinates. Choosing $\gamma$ small controls this term for all $t$ and $x$ by choosing $N$ large enough. This is independent of $d$.

The second term of (27) is handled now. Again following the argument in [5],

$$
\begin{aligned}
& P_{x}\left\{\sigma_{y_{i}^{(N)}}^{(N)} \leq t, \sigma_{y_{j}^{(N)}}^{(N)} \leq t, \sigma_{y_{i}^{(N)}}^{(N)} \leq \sigma_{y_{\jmath}^{(N)}}^{(N)}\right\} \\
& \quad=\int_{0}^{t} \int_{\partial H_{\jmath}^{(N)}} P_{x}\left\{\sigma_{y_{i}^{(N)}}^{(N)} \in d s, X_{\sigma_{y_{J}^{(N)}}^{(N)}} \in d z\right\} P_{z}\left\{\sigma_{y_{j}^{(N)}}^{(N)} \leq t-s\right\} \\
& \quad \leq P_{x}\left\{\sigma_{y_{i}^{(N)}}^{(N)} \leq t\right\} \sup _{z \in \partial H_{i}^{(N)}} P_{z}\left\{\sigma_{y_{J}^{(N)}}^{(N)} \leq t\right\} .
\end{aligned}
$$

Note the bound

$$
\int_{0}^{t} p(s, x, y) d s \leq \hat{c}|x-y|^{-1} \exp \left\{-|x-y|^{2} / 4 t\right\}
$$

for a constant $\hat{c}$. Using Lemma 1 , we see there is a constant $\hat{\hat{c}}$ independent of $x, y_{2}^{(N)}$ and $N$ with

$$
P_{x}\left\{\sigma_{y_{i}^{(N)}}^{(N)} \leq t\right\} \leq \hat{\hat{c}} N^{-1}\left|x-y_{i}^{(N)}\right|^{-1} \exp \left\{-\left|x-y_{i}^{(N)}\right|^{2} / 4 t\right\}
$$

for all $t$. Since $\left|y_{i}^{(N)}-y_{j}^{(N)}\right| \geq 3 / N$, there is a constant $\hat{C}$ with

$$
\begin{aligned}
P_{x}\left\{\begin{array}{c}
\left.\sigma_{y_{2}^{(N)}}^{(N)} \leq t, \sigma_{y_{\jmath}^{(N)}}^{(N)} \leq t, \sigma_{y_{i}^{(N)}}^{(N)} \leq \sigma_{y_{\jmath}^{(N)}}^{(N)}\right\} \\
\leq
\end{array}\right. \\
\quad \frac{\hat{C}}{N^{2}} \frac{\exp \left\{-\left|x-y_{\imath}^{(N)}\right|^{2} / 4 t\right\}}{\left|x-y_{i}^{(N)}\right|} \frac{\exp \left\{-\left|y_{i}^{(N)}-y_{\jmath}^{(N)}\right|^{2} / 4 t\right\}}{\left|y_{i}^{(N)}-y_{j}^{(N)}\right|}
\end{aligned}
$$

Now, using the definition of $\phi_{d}$ above,

$$
\begin{gathered}
\sum_{i, \jmath:\left[\left|y_{\imath}^{(N)}-y_{j}^{(N)}\right| \geq 3 / N, \quad \forall \imath \neq \jmath\right]} P_{x}\left\{\sigma_{y_{i}^{(N)}}^{(N)} \leq t, \sigma_{y_{\jmath}^{(N)}}^{(N)} \leq t\right\} \\
\leq \frac{2 \hat{C}}{N^{2}} \sum_{\imath \neq j} \frac{e^{-\left|x-y_{\jmath}^{(N)}\right|^{2} / 2 t}}{\left|x-y_{j}^{(N)}\right|} \frac{e^{-\left|y_{\imath}^{(N)}-y_{\jmath}^{(N)}\right|^{2} / 2 t}}{\left|y_{\imath}^{(N)}-y_{j}^{(N)}\right|} \phi_{d}\left(\left|x-y_{\imath}^{(N)}\right|\right) .
\end{gathered}
$$


This summation converges according to Hole Assumption III and Eq. (11),

$$
\begin{aligned}
& \frac{2 \hat{C}}{N^{2}} \sum_{\imath \neq \jmath} \frac{e^{-\left|x-y_{j}^{(N)}\right|^{2} / 2 t}}{\left|x-y_{j}^{(N)}\right|} \frac{e^{-\left|y_{i}^{(N)}-y_{j}^{(N)}\right|^{2} / 2 t}}{\left|y_{i}^{(N)}-y_{j}^{(N)}\right|} \phi_{d}\left(\left|x-y_{i}^{(N)}\right|\right) \\
& \rightarrow 2 \hat{C} \iint \frac{e^{-\left[\left(x_{1}-z_{1}\right)^{2}+\left(x_{2}-z_{2}\right)^{2}+x_{3}^{2}\right] / 2 t}}{\sqrt{\left(x_{1}-z_{1}\right)^{2}+\left(x_{2}-z_{2}\right)^{2}+x_{3}^{2}}} \frac{e^{-|y-z|^{2} / 2 t}}{|y-z|} \\
& \times \phi_{d}(|x-y|) V(y) V(z) d y d z \text {. } \\
& \leq 2 \hat{C} \iint \frac{e^{-\left[\left(x_{1}-z_{1}\right)^{2}+\left(x_{2}-z_{2}\right)^{2}+x_{3}^{2}\right] / 2 t}}{\sqrt{\left(x_{1}-z_{1}\right)^{2}+\left(x_{2}-z_{2}\right)^{2}+x_{3}^{2}}} \frac{e^{-|y-z|^{2} / 2 t}}{|y-z|} V(y) V(z) d y d z \\
& \leq 2 \hat{C} e^{-x_{3}^{2} / 2 t} \iint \frac{e^{-\left[\left(x_{1}-z_{1}\right)^{2}+\left(x_{2}-z_{2}\right)^{2}+x_{3}^{2}\right] / 2 t}}{\sqrt{\left(x_{1}-z_{1}\right)^{2}+\left(x_{2}-z_{2}\right)^{2}}} \frac{e^{-|y-z|^{2} / 2 t}}{|y-z|} V(y) V(z) d y d z .
\end{aligned}
$$

The lemma then follows by transforming the double integrals to polar coordinates, using the finite support of $V$, and using the Gaussian density formula with variance t. QED.

\section{Proof of Theorem I}

The tools are available now for a straightforward proof of Theorem I. This proof follows the general approach of [5], but the details are much more complicated.

Proof. The proof will be based on picking five values: $C_{1}, \hat{\delta}, \Delta, C_{2}$, and $N$. The value of $C_{1}$ may be chosen first, then $\hat{\delta}$, then $\Delta$, then $C_{2}$, and finally $N$. The order of these choices is critical in the following results.

Let $T_{t}, T_{t}^{(N)}$, and $T_{t}^{V}$ be the semigroups associated with Eqs. (6) and (15),

$$
\begin{aligned}
T_{t} f(x) & =E_{x}\left\{f\left(X_{t}\right)\right\}, \\
T_{t}^{(N)} f(x) & =E_{x}\left\{f\left(X_{t}\right) I_{\left(t<\sigma_{(N)}\right)}\right\}, \\
T_{t}^{V} f(x) & =E_{x}\left\{f\left(X_{t}\right) \exp \left(-2 \alpha \int_{0}^{t} V\left(X_{1_{s}}, X_{2_{s}}\right) d \zeta_{s}\right)\right\} .
\end{aligned}
$$

Define the error as

$$
I_{\varepsilon}^{(N)} \equiv \sup _{0 \leq t \leq T} \sup _{x \in D_{\varepsilon}}\left\{\left|T_{t}^{(N)} f(x)-T_{t}^{V} f(x)\right|\right\} .
$$

We use a value $0 \leq \Delta \leq T$, to be chosen later, and the integer part function \lfloor\rfloor to see that

$$
\begin{aligned}
I_{\varepsilon}^{(N)} \leq & \sup _{0 \leq t \leq T} \sup _{x \in D_{\varepsilon}}\left|\sum_{k=1}^{\lfloor t / \Delta\rfloor} T_{(k-1) \Delta}^{(N)}\left[T_{\Delta}^{(N)}-T_{\Delta}^{V}\right] T_{(\lfloor t / \Delta\rfloor-k) \Delta}^{V} f(x)\right| \\
& +\sup _{0 \leq t \leq T} \sup _{x \in D_{\varepsilon}}\left|T_{t}^{(N)} f(x)-T_{\Delta\lfloor t / \Delta\rfloor}^{(N)} f(x)\right| \\
& +\sup _{0 \leq t \leq T} \sup _{x \in D_{\varepsilon}}\left|T_{t}^{V} f(x)-T_{\Delta\lfloor t / \Delta\rfloor}^{V} f(x)\right| .
\end{aligned}
$$


Fig. 5

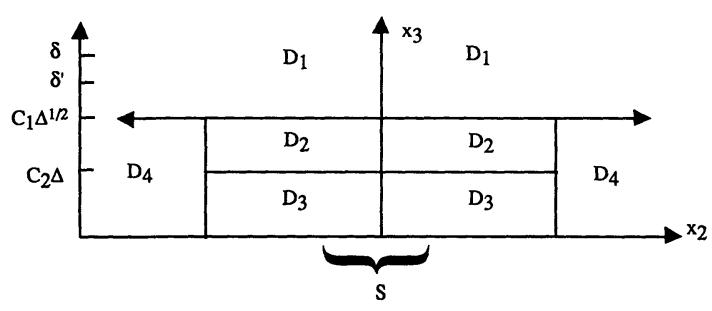

We use an indicator function to split the summation in (34). Several different sets are used to partition $\mathfrak{R} \times \mathfrak{R} \times \mathfrak{R}^{+}$. First identify a set slightly larger than $D_{\varepsilon}$. Consider a circle $\hat{C}$ in the plane $P$ with

$$
H_{i}^{(N)} \subset\left\{\left(x_{1}, x_{2}, x_{3}\right):\left(x_{1}, x_{2}\right) \in \hat{C}, x_{3}=0\right\} \text { for all } i \text { and } N
$$

and

$$
\operatorname{dist}(P-\hat{C}, S)=1 / 2
$$

$\hat{C}$ is a strict subset of $C$ in Eq. (12), implying $P-C$ is separated from $\hat{C}$ by some positive distance. Choose a thickness $\hat{\delta}$ with $\delta / 2>\hat{\delta}>0$ for $\delta$ described in the definition of $D_{\varepsilon}$ in Eq. (13). This $\hat{\delta}$ is also chosen small enough that the set

$$
\begin{aligned}
\hat{D}_{\varepsilon} \equiv\left\{\left(x_{1}, x_{2}, x_{3}\right): x_{1} \in \mathfrak{R}, x_{2} \in \mathfrak{R}, x_{3} \geq \hat{\delta}\right. \\
\left.\quad \text { if } \quad\left(x_{1}, x_{2}\right) \in \hat{C} \text { and } x_{3} \geq 0 \text { otherwise }\right\}
\end{aligned}
$$

contains $D_{\varepsilon}$ and

$$
\sup _{0 \leq t \leq T} \sup _{x \in D_{\varepsilon}} E_{x}\left\{\left(1-I_{\hat{D}_{\varepsilon}}\left(X_{t}\right)\right)\right\} \leq \varepsilon /\|f\| 40
$$

This again is just the half space minus a thin disk. Now consider, for constants $\Delta$, $C_{1}$, and $C_{2}$, with $C_{1} \Delta^{1 / 2}>C_{2} \Delta$ and $C_{1} \Delta^{1 / 2}<\hat{\delta}$,

$$
\begin{aligned}
D_{1}= & \left\{\left(x_{1}, x_{2}, x_{3}\right) \in \mathfrak{R} \times \mathfrak{R} \times \mathfrak{R}_{+}: x_{3} \geq C_{1} \Delta^{1 / 2}\right\}, \\
D_{2}= & \left(\mathfrak{R} \times \mathfrak{R} \times \mathfrak{R}^{+}-\hat{D}_{\varepsilon}\right) \\
& \cap\left\{\left(x_{1}, x_{2}, x_{3}\right) \in \mathfrak{R} \times \mathfrak{R} \times \mathfrak{R}_{+}: C_{2} \Delta \leq x_{3} \leq C_{1} \Delta^{1 / 2}\right\}, \\
D_{3}= & \left(\mathfrak{R} \times \mathfrak{R} \times \mathfrak{R}^{+}-\hat{D}_{\varepsilon}\right) \\
& \cap\left\{\left(x_{1}, x_{2}, x_{3}\right) \in \mathfrak{R} \times \mathfrak{R} \times \mathfrak{R}_{+}: 0 \leq x_{3} \leq C_{2} \Delta\right\}, \\
D_{4}= & \hat{D}_{\varepsilon} \cap\left\{\left(x_{1}, x_{2}, x_{3}\right) \in \mathfrak{R} \times \mathfrak{R} \times \mathfrak{R}_{+}: 0 \leq x_{3} \leq C_{1} \Delta^{1 / 2}\right\} .
\end{aligned}
$$

Figure 5 shows a section of the different volumes in the $\left(x_{2}, x_{3}\right)$ plane. We can choose $C_{1}>2$ large enough that

$$
E_{C_{1}}\left(\zeta_{1}^{2}\right)
$$

and

$$
C_{1} E_{C_{1}}\left(\zeta_{1}\right)
$$


are as small as needed, independent of $\hat{\delta}, \Delta, C_{2}$, and $N$. The associated processes are described in Eqs. (1) through (3). Splitting the sum,

$$
\begin{aligned}
I_{\varepsilon}^{(N)} \leq & \sum_{k=1}^{\lfloor t / \Delta\rfloor} \sup _{0 \leq t \leq T} \sup _{x \in D_{\varepsilon}}\left|T_{(k-1) \Delta}^{(N)} I_{D_{1}}\left[T_{\Delta}^{(N)}-T_{\Delta}^{V}\right] T_{(\lfloor t / \Delta\rfloor-k) \Delta}^{V} f(x)\right| \\
& +\sum_{k=1}^{\lfloor t / \Delta\rfloor} \sup _{0 \leq t \leq T} \sup _{x \in D_{\varepsilon}}\left|T_{(k-1) \Delta}^{(N)} I_{D_{2}}\left[T_{\Delta}^{(N)}-T_{\Delta}^{V}\right] T_{(\lfloor t / \Delta\rfloor-k) \Delta}^{V} f(x)\right| \\
& +\sum_{k=1}^{\lfloor t / \Delta\rfloor} \sup _{0 \leq t \leq T} \sup _{x \in D_{\varepsilon}}\left|T_{(k-1) \Delta}^{(N)} I_{D_{3}}\left[T_{\Delta}^{(N)}-T_{\Delta}^{V}\right] T_{(\lfloor t / \Delta\rfloor-k) \Delta}^{V} f(x)\right| \\
& +\sum_{k=1}^{\lfloor t / \Delta\rfloor} \sup _{0 \leq t \leq T} \sup _{x \in D_{\varepsilon}}\left|T_{(k-1) \Delta}^{(N)} I_{D_{4}}\left[T_{\Delta}^{(N)}-T_{\Delta}^{V}\right] T_{(\lfloor t / \Delta\rfloor-k) \Delta}^{V} f(x)\right| \\
& +\sup _{0 \leq t \leq T} \sup _{x \in D_{\varepsilon}}\left|T_{t}^{(N)} f(x)-T_{\Delta\lfloor t / \Delta\rfloor}^{(N)} f(x)\right| \\
& +\sup _{0 \leq t \leq T} \sup _{x \in D_{\varepsilon}}\left|T_{t}^{V} f(x)-T_{\Delta\lfloor t / \Delta\rfloor}^{V} f(x)\right| .
\end{aligned}
$$

Each of the six terms in (38) is handled separately. The first term may be estimated as

$$
\begin{aligned}
& \sup _{0 \leq t \leq T} \sup _{x \in D_{\varepsilon}}\left|T_{(k-1) \Delta}^{(N)} I_{D_{1}}\left[T_{\Delta}^{(N)}-T_{\Delta}^{V}\right] T_{(\lfloor t / \Delta\rfloor-k) \Delta}^{V} f(x)\right| \\
& \quad \leq \sup _{0 \leq t \leq T} \sup _{x \in D_{\varepsilon}} T_{(k-1) \Delta} I_{D_{1}}\left|\left[T_{\Delta}^{(N)}-T_{\Delta}^{V}\right] T_{(\lfloor t / \Delta\rfloor-k) \Delta}^{V} f(x)\right| \\
& \quad \leq \sup _{0 \leq t \leq T} \sup _{x \in D_{1}}\left|\left[T_{\Delta}^{(N)}-T_{\Delta}^{V}\right] T_{t}^{V} f(x)\right| .
\end{aligned}
$$

Terms two through four of (38) follow similarly, although more attention is paid to the indicator term. For term two,

$$
\begin{aligned}
& \sup _{0 \leq t \leq T} \sup _{x \in D_{\varepsilon}}\left|T_{(k-1) \Delta}^{(N)} I_{D_{2}}\left[T_{\Delta}^{(N)}-T_{\Delta}^{V}\right] T_{(\lfloor t / \Delta\rfloor-k) \Delta}^{V} f(x)\right| \\
& \quad \leq \sup _{0 \leq t \leq T} \sup _{x \in D_{\varepsilon}} T_{(k-1) \Delta} I_{D_{2}}\left|\left[T_{\Delta}^{(N)}-T_{\Delta}^{V}\right] T_{(\lfloor t / \Delta\rfloor-k) \Delta}^{V} f(x)\right| \\
& \quad \leq\left[\sup _{0 \leq t \leq T} \sup _{x \in D_{\varepsilon}} T_{t} I_{D_{2}}\right] \sup _{0 \leq t \leq T} \sup _{x \in D_{2}}\left|\left[T_{\Delta}^{(N)}-T_{\Delta}^{V}\right] T_{t}^{V} f(x)\right| .
\end{aligned}
$$

For term three of Eq. (38),

$$
\begin{aligned}
& \sup _{0 \leq t \leq T} \sup _{x \in D_{\varepsilon}}\left|T_{(k-1) \Delta}^{(N)} I_{D_{3}}\left[T_{\Delta}^{(N)}-T_{\Delta}^{V}\right] T_{(\lfloor t / \Delta\rfloor-k) \Delta}^{V} f(x)\right| \\
& \quad \leq \sup _{0 \leq t \leq T} \sup _{x \in D_{\varepsilon}} T_{(k-1) \Delta} I_{D_{3}}\left|\left[T_{\Delta}^{(N)}-T_{\Delta}^{V}\right] T_{(\lfloor t / \Delta\rfloor-k) \Delta}^{V} f(x)\right| \\
& \quad \leq 2\|f\| \sup _{0 \leq t \leq T} \sup _{x \in D_{\varepsilon}} T_{t} I_{D_{3}} .
\end{aligned}
$$


The fourth term of Eq. (38) may be estimated as

$$
\begin{aligned}
& \sup _{0 \leq t \leq T} \sup _{x \in D_{\varepsilon}}\left|T_{(k-1) \Delta}^{(N)} I_{D_{4}}\left[T_{\Delta}^{(N)}-T_{\Delta}^{V}\right] T_{(\lfloor t / \Delta\rfloor-k) \Delta}^{V} f(x)\right| \\
& \quad \leq \sup _{0 \leq t \leq T} \sup _{x \in D_{\varepsilon}} T_{(k-1) \Delta} I_{D_{4}}\left|\left[T_{\Delta}^{(N)}-T_{\Delta}^{V}\right] T_{(\lfloor t / \Delta\rfloor-k) \Delta}^{V} f(x)\right| \\
& \quad \leq \sup _{0 \leq t \leq T} \sup _{x \in D_{4}}\left|\left[T_{\Delta}^{(N)}-T_{\Delta}^{V}\right] T_{t}^{V} f(x)\right| .
\end{aligned}
$$

Applying Eqs. (39), (40), (41), and (42), Eq. (38) then becomes

$$
\begin{aligned}
I_{\varepsilon}^{(N)} \leq & \sup _{0 \leq t \leq T} \sup _{x \in D_{\varepsilon}}\left|T_{t}^{(N)} f(x)-T_{\Delta\lfloor t / \Delta\rfloor}^{(N)} f(x)\right| \\
& +\sup _{0 \leq t \leq T} \sup _{x \in D_{\varepsilon}}\left|T_{t}^{V} f(x)-T_{\Delta\lfloor t / \Delta\rfloor}^{V} f(x)\right| \\
& +\frac{T}{\Delta} \sup _{0 \leq t \leq T} \sup _{x \in D_{1}}\left|\left[T_{\Delta}^{(N)}-T_{\Delta}^{V}\right] T_{t}^{V} f(x)\right| \\
& +\left[\sup _{0 \leq t \leq T} \sup _{x \in D_{\varepsilon}} T_{t} I_{D_{2}}\right] \frac{T}{\Delta} \sup _{0 \leq t \leq T} \sup _{x \in D_{2}}\left|\left[T_{\Delta}^{(N)}-T_{\Delta}^{V}\right] T_{t}^{V} f(x)\right| \\
& +2 \frac{T}{\Delta}\|f\| \sup _{0 \leq t \leq T} \sup _{x \in D_{\varepsilon}} T_{t} I_{D_{3}}+\frac{T}{\Delta} \sup _{0 \leq t \leq T} \sup _{x \in D_{4}}\left|\left[T_{\Delta}^{(N)}-T_{\Delta}^{V}\right] T_{t}^{V} f(x)\right| .
\end{aligned}
$$

The first term of (43) is handled using the fact that $D_{\varepsilon}$ is separated from the holes.

$$
\begin{aligned}
& \left.\sup _{0 \leq t \leq T} \sup _{x \in D_{\varepsilon}} \mid T_{t}^{(N)} f(x)-T_{\Delta\lfloor t / \Delta\rfloor}^{(N)} f(x)\right] \mid \\
& =\sup _{0 \leq t \leq T} \sup _{x \in D_{\varepsilon}}\left|T_{\Delta\lfloor t / \Delta\rfloor}^{(N)}\left[T_{t-\Delta\lfloor t / \Delta\rfloor}^{(N)} f(x)-f(x)\right]\right| \\
& \leq \sup _{0 \leq t \leq T} \sup _{x \in D_{\varepsilon}}\left|T_{\Delta\lfloor t / \Delta\rfloor}^{(N)} I_{\hat{D}_{\varepsilon}}\left[T_{t-\Delta\lfloor t / \Delta\rfloor}^{(N)} f(x)-f(x)\right]\right| \\
& \quad+\sup _{0 \leq t \leq T} \sup _{x \in D_{\varepsilon}}\left|T_{\Delta\lfloor t / \Delta\rfloor}^{(N)}\left(1-I_{\hat{D}_{\varepsilon}}\right)\left[T_{t-\Delta\lfloor t / \Delta\rfloor}^{(N)} f(x)-f(x)\right]\right| \\
& \leq \sup _{0 \leq t \leq \Delta} \sup _{x \in \hat{D}_{\varepsilon}}\left|T_{t}^{(N)} f(x)-f(x)\right| \\
& \quad+2\|f\| \sup _{0 \leq t \leq T} \sup _{x \in D_{\varepsilon}} E_{x}\left\{\left(1-I_{\hat{D}_{\varepsilon}}\left(X_{t}\right)\right)\right\} .
\end{aligned}
$$

The second term of (44) is controlled in the definition of $\hat{D}_{\varepsilon}$. Recalling the use of $\hat{\delta}$ in the definition of $\hat{D}_{\varepsilon}$,

$$
\begin{aligned}
& \sup _{0 \leq t \leq \Delta} \sup _{x \in \hat{D}_{\varepsilon}}\left|T_{t}^{(N)} f(x)-f(x)\right| \\
& \quad \leq 2\|f\| \sup _{0 \leq t \leq \Delta} \sup _{x \in \hat{D}_{\varepsilon}} P_{x}\left\{\sup _{0 \leq s \leq t}\left|X_{3_{s}}-x_{3}\right|>\hat{\delta} / 2\right\} \\
& \quad+\sup _{0 \leq t \leq \Delta} \sup _{x \in \hat{D}_{\varepsilon}}\left|T_{t} f(x)-f(x)\right| .
\end{aligned}
$$

From the smoothness of $f$, both of these terms clearly go to zero for small $\Delta$. 
The second term of (43) follows from simple continuity.

$$
\begin{aligned}
& \sup _{0 \leq t \leq T} \sup _{x \in D_{\varepsilon}}\left|T_{t}^{V} f(x)-T_{\Delta\lfloor t / \Delta\rfloor}^{V} f(x)\right| \\
& \quad=\sup _{0 \leq t \leq T} \sup _{x \in D_{\varepsilon}}\left|T_{\Delta\lfloor t / \Delta\rfloor}^{V}\left[T_{t-\Delta\lfloor t / \Delta\rfloor}^{V} f(x)-f(x)\right]\right| \\
& \quad \leq \sup _{0 \leq t \leq \Delta} \sup _{x \in \mathfrak{R} \times \mathfrak{R} \times \mathfrak{R}^{+}}\left|T_{t}^{V} f(x)-f(x)\right| .
\end{aligned}
$$

Again this term is small for small $\Delta$ because of smoothness of $f$.

The remaining terms in (43) are really the heart of the proof, in which order of choice of constants is critical. Term three of (43), involving $D_{1}$, is examined first. Consider, to simplify notation,

$$
g(x)=T_{t}^{V} f(x)
$$

Note that $|g(x)| \rightarrow 0$ as $|x| \rightarrow \infty$. Consider for some $\Delta$,

$$
\begin{aligned}
& \frac{1}{\Delta} \sup _{x \in D_{1}}\left|T_{\Delta}^{(N)} g(x)-T_{\Delta}^{V} g(x)\right| \\
& \quad=\frac{1}{\Delta} \sup _{x \in D_{1}} \mid E_{x}\left\{g\left(X_{\Delta}\right) I_{\sigma^{(N)} \leq \Delta}\right\} \\
& \quad-E_{x}\left\{g\left(X_{\Delta}\right)\left(1-\exp \left(-2 \alpha \int_{0}^{\Delta} V\left(X_{1_{s}}, X_{2_{s}}\right) d \zeta_{s}\right)\right)\right\} .
\end{aligned}
$$

Then

$$
\begin{aligned}
& \frac{1}{\Delta} \sup _{x \in D_{1}}\left|T_{\Delta}^{(N)} g(x)-T_{\Delta}^{V} g(x)\right| \\
& \leq \frac{1}{\Delta} \sup _{x \in D_{1}}\left|E_{x}\left\{g\left(X_{\Delta}\right) I_{\sigma^{(N)} \leq \Delta}\right\}-E_{x}\left\{2 \alpha g\left(X_{\Delta}\right) \int_{0}^{\Delta} V\left(X_{1_{s}}, X_{2_{s}}\right) d \zeta_{s}\right\}\right| \\
& +\frac{1}{\Delta} \sup _{x \in D_{1}} \mid E_{x}\left\{2 \alpha g\left(X_{\Delta}\right) \int_{0}^{\Delta} V\left(X_{1_{s}}, X_{2_{s}}\right) d \zeta_{s}\right. \\
& \left.-g\left(X_{\Delta}\right)\left(1-\exp \left(-2 \alpha \int_{0}^{\Delta} V\left(X_{1_{s}}, X_{2_{s}}\right) d \zeta_{s}\right)\right)\right\} \mid \\
& \leq \frac{1}{\Delta}\|g\| \sup _{x \in D_{1}} \mid E_{x}\left\{I_{\sigma^{(N)} \leq \Delta}\right\} \\
& -E_{x}\left\{2 \alpha \int_{0}^{\Delta} V\left(X_{1_{s}}, X_{2_{s}}\right) d \zeta_{s}\right\} \mid+\frac{1}{\Delta} 4 \alpha^{2}\|g\|\|V\|^{2} \sup _{x \in D_{1}} E_{x}\left\{\zeta_{\Delta}^{2}\right\} \\
& +\frac{1}{\Delta} \sup _{x \in D_{1}}\left|E_{x}\left\{\left(g\left(X_{\Delta}\right)-g(x)\right)\left\{2 \alpha \int_{0}^{\Delta} V\left(X_{1_{s}}, X_{2_{s}}\right) d \zeta_{s}\right\}\right\}\right| \\
& +\frac{1}{\Delta} \sup _{x \in D_{1}}\left|E_{x}\left\{\left(g\left(X_{\Delta}\right)-g(x)\right) I_{\sigma^{(N)} \leq \Delta}\right\}\right| \text {. }
\end{aligned}
$$


Lemmas 3 and 5 are then applied to make the first term of (46) small. The second term of (46) follows from noting

$$
\frac{1}{\Delta} \sup _{x \in D_{1}} E_{x}\left\{\zeta_{\Delta}^{2}\right\} \leq \frac{1}{\Delta} E_{C_{1} \Delta^{1 / 2}}\left\{\zeta_{\Delta}^{2}\right\}=E_{C_{1}}\left\{\zeta_{1}^{2}\right\} .
$$

The choice of large $C_{1}$ above guarantees this is small enough, independently of $\Delta$ and $N$.

The third term follows from (47) as well as the properties of the initial function $f$. Note Eq. (45) and the smoothness of $f(x)$. There is a constant $K$ with, using the notation from Eq. (1),

$$
\begin{aligned}
E_{x}\left(\left|g\left(X_{\Delta}\right)-g(x)\right|^{2}\right) & \leq K^{2} E_{x}\left(\left|X_{\Delta}-x\right|^{2}\right) \\
& \leq 3 K^{2} E_{x}\left(\left|W_{3 t}^{x_{3}}-x_{3}\right|^{2}\right) .
\end{aligned}
$$

Now examining term three,

$$
\begin{aligned}
& \frac{1}{\Delta} \sup _{x \in D_{1}}\left|E_{x}\left\{\left(g\left(X_{\Delta}\right)-g(x)\right)\left\{2 \alpha \int_{0}^{\Delta} V\left(X_{s}\right) d \zeta_{s}\right\}\right\}\right| \\
& \leq 2 \alpha\|V\| \frac{1}{\Delta} \sup _{x \in D_{1}} E_{x}\left\{\left|g\left(X_{\Delta}\right)-g(x)\right| \zeta_{\Delta}\right\} \\
& \quad \leq 2 \alpha\|V\| \sup _{x \in D_{1}}\left(E_{x}\left\{\frac{\left|g\left(X_{\Delta}\right)-g(x)\right|^{2}}{\Delta}\right\}\right)^{1 / 2}\left(E_{x}\left\{\frac{\zeta_{\Delta}^{2}}{\Delta}\right\}\right)^{1 / 2} .
\end{aligned}
$$

Noting Eqs. (47) and (48), the first factor is bounded while the last is smaller for large $C_{1}$.

The fourth and final term of (46) requires more care. We follow the general approach described in Lemmas 1 through 3 above. Note we use $\sigma_{y_{i}^{(N)}}^{(N)}$ to indicate the hitting time of the $i^{\text {th }}$ hole of the $N^{\text {th }}$ collection centered at $y_{i}^{(N)}$. Then

$$
\begin{aligned}
& \left.\frac{1}{\Delta} \sup _{x \in D_{1}} \mid E_{x}\left\{g\left(X_{\Delta}\right)-g(x)\right) I_{\sigma^{(N)} \leq \Delta}\right\} \mid \\
& \leq \frac{1}{\Delta} \sup _{x \in D_{1}} E_{x}\left\{\left|g\left(X_{\Delta}\right)-g(x)\right| I_{\sigma^{(N)} \leq \Delta}\right\} \\
& \leq \sup _{x \in D_{1}} \frac{1}{N} \sum_{i=1}^{N} \frac{1}{\Delta} E_{x}\left\{\left|g\left(X_{\Delta}\right)-g(x)\right| N I_{\sigma_{y_{i}^{(N)}}^{(N)} \leq \Delta}\right\} \\
& \leq \sup _{x \in D_{1}} \frac{1}{N} \sum_{i=1}^{N} \frac{1}{\Delta}\left|g\left(y_{i}^{(N)}\right)-g(x)\right| E_{x}\left\{N I_{\substack{\sigma_{i}^{(N)} \\
y_{i}^{(N)}}}\right\}
\end{aligned}
$$



$$
\begin{aligned}
& +\sup _{x \in D_{1}} \frac{1}{N} \sum_{i=1}^{N} \frac{1}{\Delta} E_{x}\left\{\left|g\left(X_{\Delta}\right)-g\left(X_{\sigma_{\sigma_{\imath}^{(N)}}^{(N)}}\right)\right| N I_{\sigma_{y_{i}^{(N)}}^{(N)} \leq \Delta}\right\} \text {. }
\end{aligned}
$$


The three sums in (49) are handled in a similar way. Using the hole assumptions and Lemmas 3 and 5, for small enough $\Delta$ and then enough $N$,

$$
\begin{array}{r}
\sup _{x \in D_{1}} \frac{1}{\Delta}\left|\frac{1}{N} \sum_{i=1}^{N}\right| g\left(y_{\imath}^{(N)}\right)-g(x) \mid E_{x}\left\{N I_{\sigma_{\sigma_{\imath}^{(N)}}^{(N)} \leq \Delta}\right\} \\
-\int_{\mathfrak{R}^{2}} 2 \alpha\left(\int_{0}^{\Delta}|g(y)-g(x)| p(s, x, y) d s\right) V(y) d y \mid
\end{array}
$$

is small. Due to the smoothness of the initial condition, we again note for some constant $K$ that $|g(x)-g(y)| \leq K|x-y|$. We need only consider

$$
\begin{aligned}
\sup _{x \in D_{1}} & \frac{1}{\Delta} \int_{\mathfrak{R}^{2}} \int_{0}^{\Delta}|y-x|\left(\frac{1}{2 \pi s}\right)^{3 / 2} e^{\left\{-\frac{|x-y|^{2}}{2 s}\right\}} d s d y \\
\leq & \sup _{x \in D_{1}} \frac{1}{\Delta} \int_{\mathfrak{R}^{2}} \int_{0}^{\Delta}\left|x_{3}\right|\left(\frac{1}{2 \pi s}\right)^{3 / 2} e^{\left\{-\frac{|x-y|^{2}}{2 s}\right\}} d s d y \\
& +\sup _{x \in D_{1}} \frac{1}{\Delta} \int_{\mathfrak{R}^{2}} \int_{0}^{\Delta}\left|y_{2}-x_{2}\right|\left(\frac{1}{2 \pi s}\right)^{3 / 2} e^{\left\{-\frac{|x-y|^{2}}{2 s}\right\}} d s d y \\
& +\sup _{x \in D_{1}} \frac{1}{\Delta} \int_{\mathfrak{R}^{2}}^{\Delta}\left|y_{0}-x_{1}\right|\left(\frac{1}{2 \pi s}\right)^{3 / 2} e^{\left\{-\frac{|x-y|^{2}}{2 s}\right\}} d s d y .
\end{aligned}
$$

Note trivially that

$$
\int_{\mathfrak{R}^{2}} p_{1}\left(t, x_{1}, y_{1}\right) p_{2}\left(t, x_{2}, y_{2}\right) d y_{1} d y_{2}=1 .
$$

Then, by changing order of integration

$$
\begin{aligned}
\sup _{x \in D_{1}} & \frac{1}{\Delta} \int_{\mathfrak{R}^{2}} \int_{0}^{\Delta}\left|x_{3}\right|\left(\frac{1}{2 \pi s}\right)^{3 / 2} e^{\left\{-\frac{|x-y|^{2}}{2 s}\right\}} d s d y \\
= & \sup _{x \in D_{1}} \frac{1}{\Delta} \int_{0}^{\Delta}\left|x_{3}\right|\left(\frac{1}{2 \pi s}\right)^{1 / 2} e^{\left\{-\frac{\left|x_{3}\right|^{2}}{2 s}\right\}} \\
& \times\left[\int_{\mathfrak{R}^{2}} p_{1}\left(t, x_{1}, y_{1}\right) p_{2}\left(t, x_{2}, y_{2}\right) d y_{1} d y_{2}\right] d s \\
= & \sup _{x \in D_{1}} \frac{1}{\Delta} \int_{0}^{\Delta}\left|x_{3}\right|\left(\frac{1}{2 \pi s}\right)^{1 / 2} e^{\left\{-\frac{\left|x_{3}\right|^{2}}{2 s}\right\}} d s .
\end{aligned}
$$


Note for $0<s \leq \Delta$,

$$
\left[\sup _{x \in D_{1}}\left|x_{3}\right|\left(\frac{1}{2 \pi s}\right)^{1 / 2} e^{\left\{-\frac{\left|x_{3}\right|^{2}}{2 s}\right\}}\right]=C_{1} \Delta^{1 / 2}\left(\frac{1}{2 \pi s}\right)^{1 / 2} e^{\left\{-\frac{C_{1}^{2} \Delta}{2 s}\right\}},
$$

and also

$$
\frac{1}{\Delta^{1 / 2}} E_{C_{1} \Delta^{1 / 2}}\left\{\zeta_{\Delta}\right\}=E_{C_{1}}\left\{\zeta_{1}\right\}
$$

Applying this and change of variables, $s=\Delta \hat{s}$, and using Lemma 4 and (37), the term is small.

Treatment of the second and third terms of Eq. (50) is identical, so only the second term is described. Note, since $E(|X|) \leq E\left(|X|^{2}\right)^{1 / 2}$, again carrying through integrations,

$$
\begin{aligned}
& \int_{\mathfrak{R}^{2}} \int_{0}^{\Delta}\left|y_{2}-x_{2}\right|\left(\frac{1}{2 \pi s}\right)^{3 / 2} e^{\left\{-\frac{|x-y|^{2}}{2 s}\right\}} d s d y \\
& =\int_{\mathfrak{R}^{2}} \int_{0}^{\Delta}\left|y_{2}-x_{2}\right|\left(\frac{1}{2 \pi s}\right) e^{\left\{-\frac{|x-y|^{2}}{2 s}\right\}} e^{\left\{-\frac{\left|x_{3}\right|^{2}}{2 s}\right\}}\left[\int_{\mathfrak{R}} p_{1}\left(t, x_{1}, y_{1}\right) d y_{1}\right] d s d y_{2} \\
& =\int_{0}^{\Delta}\left(\frac{1}{2 \pi s}\right)^{1 / 2} e^{\left\{-\frac{\left|x_{3}\right|^{2}}{2 s}\right\}}\left[\int_{\mathfrak{R}}\left|y_{2}-x_{2}\right|\left(\frac{1}{2 \pi s}\right)^{1 / 2} e^{\left\{-\frac{|x-y|^{2}}{2 s}\right\}} d y_{2}\right] d s \\
& \leq \int_{0}^{\Delta} s^{1 / 2}\left(\frac{1}{2 \pi s}\right)^{1 / 2} e^{\left\{-\frac{\left|x_{3}\right|^{2}}{2 s}\right\}} d s .
\end{aligned}
$$

Because of the definition of $D_{1}$ in Eq. (36) and the requirement $C_{1}>2$, we know for $s \in[0, \Delta]$ and $x \in D_{1}$ that $s^{1 / 2} \leq C_{1} \Delta^{1 / 2} \leq\left|x_{3}\right|$. Finally,

$$
\begin{aligned}
& \sup _{x \in D_{1}} \frac{1}{\Delta} \int_{\mathfrak{R}^{2}} \int_{0}^{\Delta}\left|y_{2}-x_{2}\right|\left(\frac{1}{2 \pi s}\right)^{3 / 2} e^{\left\{-\frac{|x-y|^{2}}{2 s}\right\}} d s d y \\
& \leq \sup _{x \in D_{1}} \frac{1}{\Delta} \int_{0}^{\Delta} s^{1 / 2}\left(\frac{1}{2 \pi s}\right)^{1 / 2} e^{\left\{-\frac{\left|x_{3}\right|^{2}}{2 s}\right\}} d s \\
& \leq \sup _{x \in D_{1}} \frac{1}{\Delta} \int_{0}^{\Delta}\left|x_{3}\right|\left(\frac{1}{2 \pi s}\right)^{1 / 2} e^{\left\{-\frac{\left|x_{3}\right|^{2}}{2 s}\right\}} d s .
\end{aligned}
$$

This term is the same as the one handled immediately above in Eq. (51).

The second term in (49) follows easily by noting

$$
\left|g\left(\begin{array}{c}
X_{\sigma_{y_{2}^{(N)}}^{(N)}} \\
y_{i}
\end{array}\right)-g\left(y_{i}^{(N)}\right)\right| N I_{\sigma_{\sigma_{2}^{(N)}}^{(N)} \leq \Delta} \leq K I_{\sigma_{y_{2}^{(N)}}^{(N)} \leq \Delta},
$$


and following through the hole assumptions and lemmas again. For small enough $\Delta$ and then large enough $N$,

$$
\begin{aligned}
& \sup _{x \in D_{1}} \frac{K}{N \Delta} \mid \frac{1}{N} \sum_{i=1}^{N} E_{x}\left\{N I_{\sigma_{\sigma_{i}^{(N)}}^{(N)} \leq \Delta}\right\} \\
&-\int_{\mathfrak{R}^{2}} 2 \alpha\left(\int_{0}^{\Delta} p(s, x, y) d s\right) V(y) d y \mid
\end{aligned}
$$

is small. Again carrying through the integrals,

$$
\begin{aligned}
& \sup _{x \in D_{1}} \frac{K}{N \Delta} \int_{\mathfrak{R}^{2}} 2 \alpha\left(\int_{0}^{\Delta} p(s, x, y) d s\right) V(y) d y \\
& \leq \sup _{x \in D_{1}} \frac{2 \alpha\|V\| K}{N \Delta} \int_{0}^{\Delta}\left(\frac{1}{2 \pi s}\right)^{1 / 2} e^{\left\{-\frac{\left|x_{3}\right|^{2}}{2 s}\right\}} d s \\
& \leq \frac{2 \sqrt{2} \alpha\|V\| K}{N \sqrt{\pi \Delta}} .
\end{aligned}
$$

For fixed $\Delta$, this term is small for large enough $N$.

The third term in (49) also follows easily by using the Markov property and conditioning on the hitting time of the hole. The hole assumptions and Lemmas are then applied, along with standard inequalities and the argument in Eq. (48),

$$
\begin{aligned}
& \sup _{x \in D_{1}} \frac{1}{N} \sum_{i=1}^{N} \frac{1}{\Delta} E_{x}\left\{\left|g\left(X_{\Delta}\right)-g\left(X_{\sigma_{y_{i}^{(N)}}^{(N)}}\right)\right| N I_{\sigma_{\sigma_{i}^{(N)}}^{(N)} \leq \Delta}\right\}
\end{aligned}
$$

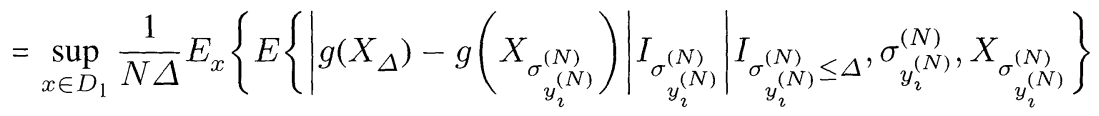

$$
\begin{aligned}
& \left.\times N I_{\sigma_{\sigma_{2}^{(N)}}^{(N)} \leq \Delta}\right\} \\
& \leq K \sup _{x \in D_{1}} \sum_{i=1}^{N} \frac{1}{N \Delta^{1 / 2}} \\
& \times E_{x}\left\{E\left\{\frac{\left|X_{\Delta}-X_{\sigma_{y_{i}^{(N)}}^{(N)}}\right|}{\Delta^{1 / 2}} I_{\sigma_{y_{i}^{(N)}}^{(N)} \leq \Delta}||_{\sigma_{y_{i}^{(N)}}^{(N)} \leq \Delta}, \sigma_{y_{i}^{(N)}}^{(N)}, X_{\sigma_{y_{i}^{(N)}}^{(N)}}\right\}\right. \\
& \left.\times N I_{\sigma_{i}^{(N)}(N)} \leq \Delta\right\} \\
& \leq K \sup _{x \in D_{1}} \sum_{\imath=1}^{N} \frac{1}{N \Delta^{1 / 2}} E_{x}\left\{N I_{\sigma_{\sigma_{\imath}}^{(N)} \leq \Delta} \leq \Delta\right\} \text {. }
\end{aligned}
$$


Again the hole assumptions and Lemmas 3 and 5 are used to show that we need to show, for small enough $\Delta$ and large enough $N$,

$$
\begin{array}{r}
\left.\sup _{x \in D_{1}} \frac{K}{\Delta^{1 / 2}} \mid \frac{1}{N} \sum_{i=1}^{N} E_{x}\left\{N I_{\substack{\sigma_{i}^{(N)} \\
y_{\imath}^{(N)}}}\right\}\right\} \\
-\int_{\mathfrak{R}^{2}} 2 \alpha\left(\int_{0}^{\Delta} p(s, x, y) d s\right) V(y) d y \mid
\end{array}
$$

is small. Again switching the order of integration and carrying through integration of the density function,

$$
\begin{aligned}
& \frac{1}{\Delta^{1 / 2}} \sup _{x \in D_{1}} \int_{\mathfrak{R}^{2}} 2 \alpha\left(\int_{0}^{\Delta} p(s, x, y) d s\right) V(y) d y \\
& \leq \frac{1}{\Delta^{1 / 2}} \sup _{x \in D_{1}} 2 \alpha\|V\| \int_{0}^{\Delta} p_{3}\left(s, x_{3}, 0\right) d s \\
& \leq \frac{1}{\Delta^{1 / 2}} 2 \alpha\|V\| \int_{0}^{\Delta} p_{3}\left(s, C_{1} \Delta^{1 / 2}, 0\right) d s .
\end{aligned}
$$

Using Lemma 4 and Eq. (52),

$$
\begin{aligned}
& \frac{1}{\Delta^{1 / 2}} \sup _{x \in D_{1}} \int_{\mathfrak{R}^{2}} 2 \alpha\left(\int_{0}^{\Delta} p(s, x, y) d s\right) V(y) d y \\
& \leq 2 \alpha\|V\| E_{C_{1}}\left\{\zeta_{1}\right\} .
\end{aligned}
$$

Consideration of Eq. (37) establishes the needed result.

We now can return to the fourth term of (43) and observe that $D_{2} \subset(\Re \times \Re \times$ $\mathfrak{R}^{+}-\hat{D}_{\varepsilon}$ ). Then, from Eq. (35), the leading coefficient

$$
\left[\sup _{0 \leq t \leq T} \sup _{x \in D_{\varepsilon}} T_{t} I_{D_{2}}\right]
$$

may be made as small as needed. The rest of the term goes to a constant, completing consideration of this term. The argument is identical to the one for term three, with the expectation that we use

$$
\frac{1}{\Delta^{1 / 2}} \sup _{x \in D_{2}} E_{x}\left\{\zeta_{\Delta}\right\} \leq \frac{1}{\Delta^{1 / 2}} E_{0}\left\{\zeta_{\Delta}\right\}=E_{0}\left\{\zeta_{1}\right\}
$$

and

$$
\frac{1}{\Delta} \sup _{x \in D_{2}} E_{x}\left\{\zeta_{\Delta}^{2}\right\} \leq \frac{1}{\Delta} E_{0}\left\{\zeta_{\Delta}^{2}\right\}=E_{0}\left\{\zeta_{1}^{2}\right\}
$$

Term five of (43) is handled by noting that, for a fixed $\Delta$, we may control the term's size by choice of $C_{2}$. 
Term six of (43) is easily controlled by recalling for the Wiener process $W_{t}$,

$$
P\left\{\max _{0 \leq s \leq \Delta} W_{s}>a\right\}=2 P\left\{W_{\Delta}>a\right\}
$$

and

$$
\frac{1}{\Delta} P\left\{\left|W_{\Delta}\right|>a\right\} \rightarrow 0 \text { for all } a \text { as } \Delta \downarrow 0
$$

The definition of $D_{4}$ explicitly separates $x \in D_{4}$ from the support of $V$ and the hole locations $\left\{y_{i}^{(N)}\right\}$. Then

$$
\frac{1}{\Delta} \sup _{x \in D_{4}} P\left\{\sigma^{(N)} \leq \Delta\right\} \rightarrow 0
$$

and

$$
\frac{1}{\Delta} \sup _{x \in D_{4}} P\left\{\int_{0}^{\Delta} V\left(X_{1_{s}}, X_{2_{s}}\right) d \zeta_{s}>0\right\} \rightarrow 0
$$

Then

$$
\begin{aligned}
& \frac{T}{\Delta} \sup _{0 \leq t \leq T} \sup _{x \in D_{4}}\left|\left[T_{\Delta}^{(N)}-T_{\Delta}^{V}\right] T_{t}^{V} f(x)\right| \\
& \leq 2\|f\| \frac{T}{\Delta} \sup _{0 \leq t \leq T} \sup _{x \in D_{4}} E_{x}\left\{I_{\sigma^{(N)} \leq \Delta}\right\} \\
& \quad+\frac{T}{\Delta} \sup _{0 \leq t \leq T} \sup _{x \in D_{4}}\left|E_{x}\left\{\left(1-\exp \left(-2 \alpha \int_{0}^{\Delta} V\left(X_{1_{s}}, X_{2_{s}}\right) d \zeta_{s}\right)\right) T_{t}^{V} f\left(X_{\Delta}\right)\right\}\right| \\
& \leq 2\|f\| \frac{T}{\Delta} \sup _{0 \leq t \leq T} \sup _{x \in D_{4}} E_{x}\left\{I_{\sigma^{(N)} \leq \Delta}\right\} \\
& \quad+2\|f\| \frac{T}{\Delta} \sup _{0 \leq t \leq T} \sup _{x \in D_{4}} P\left\{\int_{0}^{\Delta} V\left(X_{1_{s}}, X_{2_{s}}\right) d \zeta_{s}>0\right\} .
\end{aligned}
$$

The first term is managed by Eq. (53). The second follows from (54).

This finishes the proof of Theorem I. QED.

\section{Discussion of Theorem II}

Theorem II is proved using the same general approach as Theorem I. Lemma 6 provides the critical single hole limit and is discussed in the some detail. Proofs for the many hole limits as well as Theorem II are only outlined, with emphasis on differences from earlier results. 
Lemma 6. Let $T>0, \varepsilon>0, D$ be a compact set not intersecting $S$, and ${ }^{y} \zeta_{s}^{(N)}$ be the local time on the boundary of a hole of size $1 / N$ located at $y \in S$. Then

$$
E_{x}\left[N-N \exp \left(-\lambda N^{\gamma} \int_{0}^{t} d^{y} \zeta_{s}^{(N)}\right)\right] \rightarrow 2 a(\gamma) \int_{0}^{t} p(s, x, y) d s \quad \text { as } \quad N \rightarrow \infty
$$

uniformly for $x \in D, y \in S$, and $0 \leq t \leq T$. This result is independent of the rotational orientation of the hole.

Proof. Without loss of generality, assume $y=0$. Define

$$
\begin{aligned}
\tau^{(N)} & \equiv \inf \left[t:\left|X_{t}^{x}\right|=\frac{1}{N}\right] \\
X_{\tau^{(N)}} & \equiv\left\{\begin{array}{ccc}
X_{\tau^{(N)}}^{x} & \text { if } & \tau^{(N)}<\infty \\
0 & \text { if } & \tau^{(N)}=\infty
\end{array}\right\} .
\end{aligned}
$$

Then

$$
\begin{aligned}
& \lim _{N \rightarrow \infty} E_{x}\left[N-N \exp \left(-\lambda N^{\gamma} \int_{0}^{t} d^{0} \zeta_{s}^{(N)}\right)\right] \\
& =\lim _{N \rightarrow \infty} E_{x}\left[\left(N-N \exp \left(-\lambda N^{\gamma} \int_{0}^{t} d^{0} \zeta_{s}^{(N)}\right)\right) I_{\tau^{(N)} \leq t}\right] \\
& =\lim _{N \rightarrow \infty} E_{x}\left[\left(N-N \exp \left(-\lambda N^{\gamma} \int_{0}^{\infty} d^{0} \zeta_{s}^{(N)}\right)\right) I_{\tau^{(N)} \leq t}\right] \\
& \quad+\lim _{N \rightarrow \infty} E_{x}\left[N \left(\exp \left(-\lambda N^{\gamma} \int_{0}^{\infty} d^{0} \zeta_{s}^{(N)}\right)\right.\right. \\
& \left.\left.\quad-\exp \left(-\lambda N^{\gamma} \int_{0}^{t} d^{0} \zeta_{s}^{(N)}\right)\right) I_{\tau^{(N)} \leq t}\right]
\end{aligned}
$$

We follow the general pattern of the proof of Lemma One. Again the approach is illustrated in Fig. 3. A random path is followed until it (possibly) intersects a sphere of radius $1 / n$. For term one of (55),

$$
\begin{gathered}
\lim _{N \rightarrow \infty} E_{x}\left[\left(N-N \exp \left(-\lambda N^{\gamma} \int_{0}^{\infty} d^{0} \zeta_{s}^{(N)}\right)\right) I_{\tau^{(N)} \leq t}\right] \\
=\lim _{N \rightarrow \infty} E_{x}\left[N I _ { \tau ^ { ( N ) } \leq t } E \left(1-\exp \left(-\lambda N^{\gamma}\right.\right.\right. \\
\left.\quad \times \int_{0}^{\infty} d^{0} \zeta_{s}^{(N)} I_{\tau^{(N)} \leq t}\right) \mid X_{\left.\left.\tau^{(N)}, \tau^{(N)}, I_{\tau^{(N)}<\infty}\right)\right] .}
\end{gathered}
$$


For large $N$, the distribution $X_{\tau^{(N)}}$, conditioned on $\tau^{(N)}<\infty$, converges to uniform on the half sphere of radius $1 / N$. The conditional expectation is examined closely by considering an equivalent representation. Consider the solution $w$ of the equation

$$
\begin{aligned}
& \frac{\partial w^{N}}{\partial t}=\frac{1}{2} \Delta w^{N}(x, t) \quad t \geq 0, \quad x \in \mathfrak{R} \times \mathfrak{R} \times \mathfrak{R}^{+}, \\
& w^{N}(x, 0)=1, \\
& \frac{\partial w^{N}}{\partial n}(x, t)=0 \text {, } \\
& \text { for } x \in \partial P-H^{N}, \quad \text { and } n \text { the outward normal } \\
& \frac{\partial w^{N}}{\partial n}(x, t)-\lambda N^{\gamma} w^{N}(x, t)=0 \\
& \text { for } x \in \partial H^{N}, \quad \text { and } n \text { the outward normal, }
\end{aligned}
$$

or

$$
w^{N}(x, t)=E_{x}\left\{\exp \left(-\lambda N^{\gamma} \int_{0}^{t} d^{0} \zeta_{s}^{(N)}\right)\right\} .
$$

Define

$$
\hat{w}^{N}=1-w^{N}
$$

Using Eq. (57), we see

$$
\begin{gathered}
\begin{array}{c}
\frac{\partial \hat{w}^{N}}{\partial t}=\frac{1}{2} \Delta \hat{w}^{N}(x, t) \quad t \geq 0, \quad x \in \mathfrak{R} \times \mathfrak{R} \times \mathfrak{R}^{+}, \\
\hat{w}^{N}(x, 0)=0, \\
\frac{\partial \hat{w}^{N}}{\partial n}(x, t)=0,
\end{array} \\
\text { for } x \in \partial P-H^{N}, \quad \text { and } n \text { the outward normal } \\
\quad \frac{\partial \hat{w}^{N}}{\partial n}(x, t)-\lambda N^{\gamma} \hat{w}^{N}(x, t)=-\lambda N^{\gamma} \\
\text { for } \quad x \in \partial H^{N}, \quad \text { and } n \text { the outward normal, }
\end{gathered}
$$

Now we use the definition of $a(\gamma)$. This is accomplished by rescaling with $\hat{t}=N^{2} t$ and $\hat{x}=N x$. Then, using Eq. (56) and letting $t \rightarrow \infty$,

$$
\begin{aligned}
& \lim _{N \rightarrow \infty} E_{x}\left[\left(N-N \exp \left(-\lambda N^{\gamma} \int_{0}^{\infty} d^{0} \zeta_{s}^{(N)}\right)\right) I_{\tau^{(N)} \leq t}\right] \\
& =2 a(\gamma) \int_{0}^{t} p(s, x, 0) d s .
\end{aligned}
$$

Again we have used Lemma 1 in [5], whose result is described in Eq. (22). 
The second term of Eq. (55) follows from the discussion relating to Eq. (57) by noting, for some $\Delta>0$ to be chosen later,

$$
\begin{aligned}
& \lim _{N \rightarrow \infty} E_{x}\left[N \left(\exp \left(-\lambda N^{\gamma} \int_{0}^{t} d^{0} \zeta_{s}^{(N)}\right)\right.\right. \\
& \left.\left.\quad-\exp \left(-\lambda N^{\gamma} \int_{0}^{\infty} d^{0} \zeta_{s}^{(N)}\right)\right) I_{\tau^{(N)} \leq t}\right] \\
& \leq \lim _{N \rightarrow \infty} E_{x}\left[N I_{t-\Delta<\tau^{(N)} \leq t}\right] \\
& \quad+\lim _{N \rightarrow \infty} E_{x}\left[N I_{\tau^{(N)} \leq t-\Delta} E\right]\left(\exp \left(-\lambda N^{\gamma} \int_{0}^{t} d^{0} \zeta_{s}^{(N)}\right)\right. \\
& \left.\left.\left.\quad-\exp \left(-\lambda N^{\gamma} \int_{0}^{\infty} d^{0} \zeta_{s}^{(N)}\right)\right) \mid X_{\tau^{(N)}}, \tau^{(N)}, I_{\tau^{(N)}<t-\Delta}\right]\right]
\end{aligned}
$$

Equation (22) is used for the first term, which is made small by choice of $\Delta$ and $N$. To address the second term, again use the representation in Eq. (57) and then rescale with $\hat{t}=N^{2} t$ and $\hat{x}=N x$. This completes the proof of Lemma 6. QED.

Lemma 7 provides a bound for the many hole situation, and is similar to Lemma 5 for the Dirichlet condition.

Lemma 7. Given $\varepsilon>0, T<\infty$, and closed $D \subset \mathfrak{R} \times \mathfrak{R} \times \mathfrak{R}^{+}$not intersecting $S$, there exists an $N_{0}(\varepsilon, T)$ with the following. Let

$$
d=\frac{1}{2} \operatorname{distance}(D, S)
$$

For all $N \geq N_{0}, x \in D$, and $0 \leq t \leq T$,

$$
E_{x}\left\{1-\exp \left(-\lambda N^{\gamma} \int_{0}^{t} d \zeta_{s}^{(N)}\right)\right\}>2 E_{x}\left[a(\gamma) \int_{0}^{t} V\left(X_{s}^{1}, X_{s}^{2}\right) d \zeta_{s}\right]-\varepsilon-g(t)
$$

with

$$
g(t)=t C \exp \left(\frac{-d}{2 t}\right) \text { for a constant } C
$$

The constant $C$ is independent of $N, \varepsilon, D$, and $T$.

Outline of Proof. Let $\varepsilon>0$ and fix $\delta$, to be chosen later. First consider contributions from holes that are adequately far apart. We sum over all holes that are at least $3 / N$ from their nearest neighbor. Recall the local time notation in Eqs. (4) and (5). Again 
use $\sigma_{y_{i}^{(N)}}^{(N)}$ as the hole hitting time. Then

$$
\begin{aligned}
& E_{x}\left\{1-\exp \left(-\lambda N^{\gamma} \int_{0}^{t} d \zeta_{s}^{(N)}\right)\right\} \\
& \geq E_{x}\left\{\sum_{i:\left|y_{2}^{(N)}-y_{\jmath}^{(N)}\right| \geq 3 / N \forall \jmath \neq i} I_{\sigma_{y_{2}^{(N)}}^{(N)} \leq t, \sigma_{y_{j}^{(N)}}^{(N)}>t \forall j \neq i}\right. \\
& \left.\quad\left(1-\exp \left(-\lambda N^{\gamma} \int_{0}^{t} d \zeta_{s}^{(N)}\right)\right)\right\} .
\end{aligned}
$$

Because the indicator restricts contributions to trajectories interacting with only one hole,

$$
\begin{aligned}
& E_{x}\left\{1-\exp \left(-\lambda N^{\gamma} \int_{0}^{t} d \zeta_{s}^{(N)}\right)\right\} \\
& \quad \geq E_{x}\left\{\sum_{i:\left|y_{2}^{(N)}-y_{\jmath}^{(N)}\right| \geq 3 / N \quad \forall j \neq i}^{N} I_{\sigma_{y_{2}^{(N)}}^{(N)} \leq t, \sigma_{y_{j}^{(N)}}^{(N)}>t \forall j \neq i}\right. \\
& \left.\left(1-\exp \left(-\lambda N^{\gamma} \int_{0}^{t} d^{i} \zeta_{s}^{(N)}\right)\right)\right\} .
\end{aligned}
$$

Then

$$
\begin{aligned}
E_{x}\left\{1-\exp \left(-\lambda N^{\gamma} \int_{0}^{t} d \zeta_{s}^{(N)}\right)\right\} \\
\geq E_{x}\left\{\sum_{i:\left|y_{\imath}^{(N)}-y_{j}^{(N)}\right| \geq 3 / N \forall j \neq \imath}^{N}\right. \\
\\
\left.\quad\left(1-\exp \left(-\lambda N^{\gamma} \int_{0}^{t} d^{\imath} \zeta_{s}^{(N)}\right)\right)\right\} \\
\quad-E_{x}\left\{\sum_{i:\left|y_{\imath}^{(N)}-y_{j}^{(N)}\right| \geq 3 / N \forall j \neq \imath}^{N}\left(1-I_{\sigma_{y_{i}^{(N)}}^{(N)} \leq t, \sigma_{y_{j}^{(N)}}^{(N)}>t \forall j \neq i}\right)\right. \\
\\
\left.\quad\left(1-\exp \left(-\lambda N^{\gamma} \int_{0}^{t} d^{\imath} \zeta_{s}^{(N)}\right)\right)\right\} .
\end{aligned}
$$


We address the first term in (58) first.

$$
\begin{aligned}
& E_{x}\left\{\sum_{i:\left|y_{i}^{(N)}-y_{\jmath}^{(N)}\right|_{\geq 3 / N}^{N} \forall j \neq i}\left(1-\exp \left(-\lambda N^{\gamma} \int_{0}^{t} d^{i} \zeta_{s}^{(N)}\right)\right)\right\} \\
& =E_{x}\left\{\sum_{i=1}^{N}\left(1-\exp \left(-\lambda N^{\gamma} \int_{0}^{t} d^{2} \zeta_{s}^{(N)}\right)\right)\right\} \\
& -E_{x}\left\{\sum_{i:\left|y_{2}^{(N)}-y_{J}^{(N)}\right|<3 / N \quad \forall j \neq \imath}^{N}\left(1-\exp \left(-\lambda N^{\gamma} \int_{0}^{t} d^{i} \zeta_{s}^{(N)}\right)\right)\right\} .
\end{aligned}
$$

Our first term is handled exactly as discussed in Eqs. (25) through (26), by application of Lemmas 4 and 6 and the Hole Assumptions. This establishes the bound in the lemma statement.

For the second term of Eq. (59),

$$
E_{x}\left\{1-\exp \left(-\lambda N^{\gamma} \int_{0}^{t} d^{\imath} \zeta_{s}^{(N)}\right)\right\} \leq P_{x}\left\{\sigma_{y_{\imath}^{(N)}}^{(N)} \leq t\right\}
$$

The discussion around Eqs. (30) and (31) establish the size of this term.

Proof of the lemma will be complete with examination of the second term in Eq. (58). This is the step where the function $g$ will be defined. Note we replace a single summation in $i$ with a double summation in $i$ and $j$,

$$
\begin{aligned}
& E_{x}\left\{\sum_{i:\left|y_{\imath}^{(N)}-y_{j}^{(N)}\right| \geq 3 / N \quad \forall j \neq i}^{N} I_{\sigma_{\sigma_{i}^{(N)}}^{(N)} \leq t, \sigma_{y_{j}^{(N)}}^{(N)} \leq t \text { for some } j \neq i}\right. \\
& \left.\left(1-\exp \left(-\lambda N^{\gamma} \int_{0}^{t} d^{i} \zeta_{s}^{(N)}\right)\right)\right\} \\
& \leq E_{x}\left\{\sum_{i, \jmath:\left|y_{i}^{(N)}-y_{j}^{(N)}\right| \geq 3 / N}^{N} \underset{\forall j \neq i}{I_{\sigma_{i}^{(N)} \leq(N)}^{(N)} \leq t, \sigma_{y_{j}^{(N)}}^{(N)} \leq t}\right. \\
& \left.\left(1-\exp \left(-\lambda N^{\gamma} \int_{0}^{t} d^{i} \zeta_{s}^{(N)}\right)\right)\right\}
\end{aligned}
$$

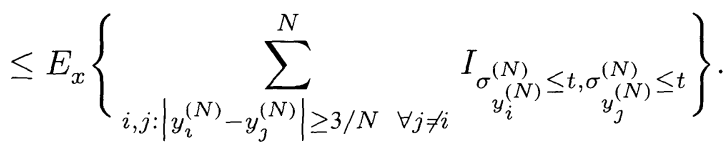

This is the same term we have in Eqs. (32) through (33). QED 
Lemma 8 provides the bound in the other direction, and its proof closely resembles the proof of Lemma 3.

Lemma 8. Given $\varepsilon>0, T<\infty$, and closed $D \subset \mathfrak{R} \times \mathfrak{R} \times \mathfrak{R}^{+}$not intersecting $S$, there exists an $N_{0}(\varepsilon, T)$ with the following. For all

$$
\begin{gathered}
N \geq N_{0}, \quad x \in D, \quad \text { and } \quad 0 \leq t \leq T \\
E_{x}\left\{1-\exp \left(-\lambda N^{\gamma} \int_{0}^{t} d \zeta_{s}^{(N)}\right)\right\}<2 E_{x}\left[a(\gamma) \int_{0}^{t} V\left(X_{s}^{1}, X_{s}^{2}\right) d \zeta_{s}\right]+\varepsilon
\end{gathered}
$$

with

$$
\sigma^{(N)} \equiv\left\{\inf t: X_{t}^{x} \in \bigcup_{i=1}^{N} \partial H_{i}^{(N)}\right\}
$$

Outline of Proof. Note for constants $a>0$ and $b>0$,

$$
1-e^{-a-b} \leq\left(1-e^{-a}\right)+\left(1-e^{-b}\right)
$$

Recalling the definition of the local times,

$$
\begin{aligned}
& E_{x}\left\{1-\exp \left(-\lambda N^{\gamma} \int_{0}^{t} d \zeta_{s}^{(N)}\right)\right\} \\
& \leq E_{x}\left\{1-\exp \left(-\lambda N^{\gamma} \sum_{i=1}^{N} \int_{0}^{t} d^{2} \zeta_{s}^{(N)}\right)\right\} \\
& \leq \sum_{i=1}^{N} E_{x}\left\{1-\exp \left(-\lambda N^{\gamma} \int_{0}^{t} d^{2} \zeta_{s}^{(N)}\right)\right\} .
\end{aligned}
$$

Again this term is handled in Eq. (59). QED.

Lemmas 7 and 8 provide the tools for proving Theorem II. For $a(\gamma)>0$, the proof is identical to that of Theorem I, with the expression $\left(1-\exp \left(-\lambda N^{\gamma} \zeta_{\Delta}^{(N)}\right)\right)$ replacing $I_{\sigma^{N}<\Delta}$ in the equations. For $a(\gamma)=0$, the argument is even simpler and all the required terms are examined above.

\section{References}

1. Del Vecchio, T.: The thick Neumann's sieve. J. Fac. of Sci. of the Univ. Tokyo 36, (1989)

2. Murat, F.: The Neumann sieve. Nonlinear variational problems. Marino, A. et. al. (eds.) London: Pitman, 1985

3. Kac, M.: Probabilistic methods in some problems of scattering theory. Rocky Mountain J. Math. 4, no. 3, (1974)

4. Rauch, J., Taylor, M.: Potential and scattering theory on wildly perturbed domains. Funct. Anal. 18, 27-59 (1975) 
5. Papanicolaou, G., Varadhan, S.: Diffusions in regions with many small holes. Stochastic differential systems filtering and control. Proceedings of the IFIP-WG 7/1 Working Conference, Vilnius, Lithuania, Aug. Aug. 28-Sept. 2, 1978

6. Figari, R., Orlandi, E., Teta, S.: The Laplacian in regions with many small obstacles: fluctuations around the limit operator. Stat. Phys. 41, Nos. 3/4, 465-487 (1985)

7. Khruslov, E.I., Marchenko, V.A.: Boundary value problems in regions with fine-grained boundaries. Kiev: Naukova Dumka, 1974

8. Weiryb, S.: Etude Asymptotique par des Mesures de $\mathbb{R}^{3}$ de Saucisses de Wiener Localisses. Probability Theory and Related Fields 73, no. 1, 135-148, (1986)

Communicated by M.E. Fisher 